\title{
Dust in brown dwarfs
}

\section{Dust formation under turbulent conditions on microscopic scales}

\author{
Ch. Helling ${ }^{1}$, M. Oevermann ${ }^{2}$, M. J. H. Lüttke ${ }^{1}$, R. Klein ${ }^{2,3}$, and E. Sedlmayr ${ }^{1}$ \\ 1 Zentrum für Astronomie und Astrophysik, Technische Universität Berlin, Hardenbergstraße 36, \\ 10623 Berlin, Germany \\ 2 Konrad-Zuse-Zenrum für Informationstechnik Berlin, Takustraße 7, 14195 Berlin, Germany \\ 3 Fachbereich Mathematik und Informatik, Freie Universität Berlin, Takustraße 7, 14195 Berlin, Germany
}

Received 3 May 2001 / Accepted 27 June 2001

\begin{abstract}
Dust formation in turbulent media is studied adopting the example of brown dwarf atmospheres. By combining asymptotic techniques and time-dependent, multi-dimensional numerical simulations, we show that acoustic waves originating from convective motions provide a mechanism to initiate dust nucleation in otherwise dust-hostile environments. The subsequently growing particles cause a radiative cooling strong enough to reinitiate efficient dust formation resulting in a strongly inhomogeneous distribution of dust in such environments. Recent observations bear indications for such cloudy dust distributions in brown dwarf atmospheres.
\end{abstract}

\section{Introduction}

Brown dwarfs are star-like objects with masses below the stable hydrogen burning limit $\left(\approx 7.5 \% M_{\odot}\right.$ for solar metallicity $Z_{\odot}$; see Chabrier \& Baraffe 2000) and electron degeneracy balances the gravitational collapse keeping the interior in hydrostatic equilibrium. The objects are very faint and the radiation pressure is negligible in their atmospheres. The interior of brown dwarfs is entirely convective. Chabrier \& Baraffe (1997) found the upper limit of fully convective stars to be $0.35 M_{\odot}\left(10^{-2} \lesssim Z / Z_{\odot} \lesssim 1\right)$ based on the mixing length theory. The top of the interior's convective zone lies only a few pressure scale heights $\left(H_{\mathrm{p}}\right)$ below the photosphere (Chabrier et al. 2000). The convective transport of energy, momentum, and material reaches into the brown dwarf atmosphere (Chabrier \& Baraffe 1997; Burrows et al. 1997; Burrows \& Sharp 1999; Allard et al. 1999; Tsuji et al. 1999) and can be important for the replenishment of the atmosphere by heavy elements which might have been previously depleted by incorporation into dust particles and their gravitational settling (Chabrier et al. 2000; Helling et al. 2000).

Indeed, the $\mathrm{CO}$ molecule has been observed in the brown dwarf Gl 229B in atmospheric regions too cool for $\mathrm{CO}$ to be present according to chemical equilibrium calculations (Noll et al. 1997; Oppenheimer et al. 1998). The cause may be: i) Hot, $\mathrm{CO}$ containing gas reaches into the atmosphere due to convective material transport. The CO molecule is not destroyed during the subsequent

Send offprint requests to: Ch. Helling,

e-mail: chris@astro.physik.tu-berlin.de cooling since the chemical composition is determined by non-equilibrium effects; ii) convection transports warm, $\mathrm{CO}$ containing gas into the cooler atmosphere and a pattern of warm and dense regions results. Therefore the CO molecule sustains inside the warm regions while chemical equilibrium holds; or iii) The interstellar UV field is strong enough to initiate in situ CO formation by photo reactions. However, the chemical non-equilibrium hypothesis (i) is rather unlikely due to the high density of the atmospheric gas (see e.g. Patzer et al. 1999b) and only the last possibility (iii) can omit the presents of convective energy transport. Oppenheimer et al. (1998) suggested also that the observation of the atomic Cs line in Gl 229B (also GD 165B) might indicate the existence of an convective material transport since $\mathrm{Cs}$ should be bound in some molecules at these low temperatures according to chemical equilibrium arguments.

Furthermore, according to estimates based on static model atmospheres, the Reynolds number - a measure of the viscous damping capability of the gas - exceeds unity by orders of magnitude already on microscopic scales ( $l_{\text {ref }}<H_{\rho}, H_{\rho}$ density scale height). Consequently, disturbances e.g. introduced by the interior convective overshooting into the atmosphere can not be damped efficiently. Therefore, a brown dwarf atmosphere may be expected to consist of a turbulent fluid field fed by the interior's convection which also may influence the distribution of a possible solid component.

Bailer-Jones \& Mundt (2001a,b; see also Bailer-Jones \& Mundt 1999) found 11 of a sample of 21 brown dwarfs (see their Table 2) to be variable on time scales of a few 
minutes and to a few tens of hours with a confidence level of $99 \%$. Eight of these show small amplitude variations between 0.01 and 0.03 mag not correlated with a rotation period. It was suggested by the authors that these variations might well result from an inhomogeneous distribution of dust in the atmosphere. Martín et al. (2001) follow this interpretation for their observations of the ultracool, very inactive dwarf BRI 0021-0214. Oppenheimer et al. (1998) found evidence for variability in the brown dwarf Gl 229B of a maximum of $10 \%$ on time scales from several minutes over a few days to one year in the $1.6 \mu \mathrm{m} \mathrm{CH}_{4}$ absorption line. Gelino et al. (2001) argue that magnetic spots are unlikely to be responsible for such variability since the magnetic Reynolds number of the atmospheric gas is very small on the mesoscopic scales $\left(l_{\text {ref }}=H_{\mathrm{P}}\right)$ considered.

Brown Dwarf atmospheres provide favorable conditions for the gas-solid phase transition due to their low temperatures and high densities. Stevenson (1986) and Lunine et al. (1989) were the first to point out the importance of dust as opacity source in the brown dwarf's evolution. Tsuji et al. (1996b) suggested dust containing photospheres $\left(T_{\text {eff }}=1800 \mathrm{~K}\right)$ as the only reasonable fit to the observed spectral energy distribution (SED) of the brown dwarf GD 165B observed by Tinney et al. (1993) and to the infrared $\mathrm{H}_{2} \mathrm{O}$ bands observed by Jones et al. (1994). Kirkpatrick et al. (1999a) provide a comparable fit by static model atmospheres considering the existence of power-law-sized solid particles in order to account for the unexpectedly weak molecular absorption bands. But still, too strong $\mathrm{VO}$ and $\mathrm{TiO}$ bands are predicted from the models.

Liebert et al. (2000) argue that a correct treatment of the line wings (more advanced than Lorenzian profile) of the alkali absorption lines ( $\mathrm{Na}$ I and K I doublets) could make the necessity to include dust in brown dwarfs atmospheres obsolete.

The present interpretation of the observed brown dwarf spectra relies exclusively on static model atmospheres with elaborate treatments of frequency dependent radiative transport including a large body of atomic and molecular line data. Encouraged by extensive theoretical investigations of possible compounds in the cool and dense environment of brown dwarfs (and gas planets; Burrows \& Sharp 1999; Lodders 1999), dust has been included in the static model atmospheres as an opacity source. The dust caused a strong backwarming and element depletion, resulting in weaker molecular absorption (Tsuji et al. 1996a, 1996b; Jones \& Tsuji 1997; Pavlenko et al. 2000; Chabrier et al. 2000; Leinert et al. 2000; Basri et al. 2000; Allard et al. 2001) and a better agreement with observation could be achieved. Depending on the purpose of the models, dust has been taken out or taken into account as opacity source. The easy access of the dust complex in the static model atmospheres must be payed for by various simplifications:

- The compounds are assumed to be in phase equilibrium with the gas (e.g. Burrows \& Sharp 1999; Chabrier et al. 2000; Leinert et al. 2000; Allard et al. 2001) as introduced by Grossmann (1972) and Lunine et al. (1989).
If, however, the supersaturation ratio $S$ equals 1 (phase equilibrium), and therefore the formation and destruction rates equilibrate, no solid particle can form. A sufficient supersaturation is needed to form non-plane solids, therefore, a much lower temperature than $T(S=1)$ is required (see e.g. Rossow 1978; Gail et al. 1984).

- Adopting phase equilibrium, the description of the formation of the compounds is omitted. Therefore it appears the inconsistence that the monomer molecules from which the compounds are meant to form are often not present in the gas phase or only in very small amounts (e.g. $\mathrm{Al}_{2} \mathrm{O}_{3}, \mathrm{CaTiO}_{3}, \mathrm{MgAl}_{2} \mathrm{O}_{4}, \mathrm{CaMgSi}_{2} \mathrm{O}_{7} \ldots$...).

- The grain size distribution had to be prescribed. The interstellar power law has been adopted for grain sizes of $0.025 \ldots 0.25 \mu \mathrm{m}$ in order to derive the spectral characteristics of the dust for the opacity calculation.

Lunine et al. (1989) were the first to point out the influence of the grain size on the resulting grey atmosphere structure in brown dwarfs following time-scale arguments of Rossow (1978).

The gas-solid phase transition is in fact a nonequilibrium process and the grain size distribution is not known a priori. Furthermore, the formation of dust particles is a time dependent process and a consistent treatment with hydro- and thermodynamics is therefore required. One example, where the implications of such a consistent treatment due to non-linear interactions have been shown by numerical simulations, are circumstellar dust shells of long periodic variables (Gail \& Sedlmayr 1987; Dominik et al. 1990; Fleischer et al. 1992; Höfner et al. 1994; Helling et al. 2000; Winters et al. 2000). Here, the non-linear coupling between dust and hydro-/thermodynamics causes the creation of dust induced shock waves due to the high luminosity of the star and the large opacity of the dust, the occurrence of an onion-like distribution of dust around the star due to backwarming, and in a time dependent size distribution of the dust grains due to time-dependent thermodynamic conditions.

In brown dwarfs, dust-free convective cells originating from the interior convective zone travel upward and decay into smaller and smaller eddies at some point in the atmosphere. Following Kolmogoroff's assumption (e.g. Frisch 1995), energy is transfered from the largest scales, comparable to the system's size, by non-linear processes into smaller and smaller scales until the energy is finally dissipated on the Kolmogoroff scale ${ }^{1}$ by the viscosity of the gas. The smaller the scale, the smaller the influence of large scale flow structures. Then, the resulting turbulent fluctuations can be considered homogeneous and isotropic on microscopic scales. Considering that the large scale turbulent motions have a characteristic Mach number of the order of unity, resulting acoustic waves (Fig. 1, l.h.s.) create a turbulent temperature and density field in the

\footnotetext{
${ }^{1}$ Kolmogoroff scale $\eta=\frac{L}{R e^{3 / 4}}$, with the integral scale $L=$ $l_{\text {ref }}=10^{4} \mathrm{~cm}$ and $R e=10^{8}$ (see Table 2.1$) \Rightarrow \eta=10^{-2} \mathrm{~cm}$. For comparison, the mean free path is $10^{-3} \mathrm{~cm}$.
} 
atmosphere which influences the dust complex due to its high temperature and density sensitivity. Indications for such a turbulent atmosphere may be the observations of CO (and possibly Cs) in Gl 229B (Oppenheimer et al. 1998) but also the non-periodic variability observed by Bailer-Jones \& Mundt (2001a,b) and Martín et al. (2001). The aim of this paper is therefore to study the complex, non-linear interactions between dust formation, chemistry and turbulence at first on microscopic scales.

Our approach is to combine asymptotic techniques and numerical simulations. The asymptotic techniques will allow us to consider the long term behavior of the dust forming system by the use of a reduced model. It also serves as an independent method to test our numerical results. The numerical simulations of the full model problem are performed in order to consistently study dust formation in a strongly fluctuating environment.

In Sect. 2, the full model problem containing hydro-, thermodynamics and the dust complex is outlined. Typical time scales and the characteristic numbers of the system - the brown dwarf atmosphere - are discussed in order to justify our model approach.

Section 3 contains the asymptotic analysis of a reduced model problem and the study of the long term behavior of the dust complex for a typical brown dwarf atmosphere situation.

Section 4 starts with the validation of the multidimensional code on the basis of a zero dimensional test problem. In two scenarios, $1 \mathrm{D}$ and $2 \mathrm{D}$, the appearance of an intermittent dust distribution caused by acousticsinduced thermodynamic fluctuations in the microscopic regime of the turbulent brown dwarf atmosphere is shown.

In Sect. 5 we discuss the problem of the grain size distribution, the gap between $T(S=1)$ and the threshold temperature for efficient nucleation, the possible rain out, the problem of cloud formation in the framework of our studies and the site of the dust in the atmosphere.

Section 6 contains our conclusions.

\section{The model problem}

The model problem is twofold:

Complex A: The hydro- and thermodynamics which are described following the classical approach for an inviscid, compressible fluid.

Complex B: The chemistry and dust formation. The dust formation is a two step process - nucleation and growth (Rossow 1978; Gail et al. 1984; Gail \& Sedlmayr 1988) - and depends by the amount of condensible species on the local temperature and density of the gas which are determined by Complex A. Dust formation is initiated by the clustering of molecular species from the gas phase into clusters consisting of roughly 100 molecules on which irreversible growth of gas phase species becomes possible. Since aggregates of this size are usually less tightly bound than the bulk condensate, considerable supercooling below the stability limit of the corresponding solid material is required before the seed nuclei can form. These nuclei serve as centers for subsequent growth to macroscopic sizes. The material initiating dust formation does not need to be identical to the main dust material formed during subsequent growth on the initial seed nuclei, since even a rare component of the gas mixture can provide the required seed nuclei if these are formed at higher temperatures than all other material from which seed nuclei might be formed.

The description of the dust nucleation is based on the modified classical nucleation theory. The dust growth is described by the momentum method (Gail \& Sedlmayr 1986, 1988; Dominik et al. 1993) in combination with the differential equations describing the element conservation.

\subsection{Model equations}

The model equations have been transformed into their dimensionless form by substituting $\rho \rightarrow \rho / \rho_{\text {ref }}, \boldsymbol{v} \rightarrow \boldsymbol{v} / v_{\text {ref }}$, $P \rightarrow P / P_{\mathrm{ref}}, T \rightarrow T / T_{\mathrm{ref}}, l \rightarrow l / l_{\mathrm{ref}}, t \rightarrow t / t_{\mathrm{ref}}$ $\left(t_{\text {ref }}=l_{\text {ref }} / v_{\text {ref }}\right), \boldsymbol{g} \rightarrow \boldsymbol{g} / g_{\text {ref }}, \kappa \rightarrow \kappa / \kappa_{\text {ref }}, J_{*} \rightarrow J_{*} / J_{* \text {,ref }}$, $L_{j} \rightarrow L_{j} / L_{j, \text { ref }}, \chi^{\text {net }} \rightarrow \chi^{\text {net }} / \chi_{\text {ref }}, \epsilon_{x} \rightarrow \epsilon_{x} / \epsilon_{\text {ref }}$ and dimensionless numbers have been identified. (All symbols will be explained shortly.)

\section{Complex A:}

The equations of mass, momentum, and energy conservation in dimensionless form read:

$$
\begin{aligned}
& (\rho)_{\mathrm{t}}+\nabla \cdot(\rho \boldsymbol{v})=0 \\
& (\rho \boldsymbol{v})_{\mathrm{t}}+\nabla \cdot(\rho \boldsymbol{v} \circ \boldsymbol{v})=-\frac{1}{\gamma M^{2}} \nabla P-\frac{\gamma M^{2}}{F r} \rho \boldsymbol{g} \\
& (\rho e)_{\mathrm{t}}+\nabla \cdot(\boldsymbol{v}[\rho e+P])=R d \kappa\left(T_{\mathrm{RE}}^{4}-T^{4}\right)
\end{aligned}
$$

with $\rho$ being the dimensionless gas density, $P$ the dimensionless gas pressure, $\boldsymbol{v}$ the dimensionless hydrodynamic velocity vector with $\boldsymbol{v} \circ \boldsymbol{v}$ the tensor product, $T$ the dimensionless temperature, $T_{\mathrm{RE}}$ the dimensionless radiative equilibrium temperature and $\kappa$ the dimensionless total absorption coefficient. For $M, F r$, and $R d$ see Sect. 2.2. Assuming local thermal equilibrium, the interaction between matter and radiation field can be described by a relaxation ansatz on the r.h.s. of the energy equation (Eq. (3)). $e$ is the total energy of the gas given by the sum of kinetic, potential and thermal energy with $\boldsymbol{g}$ the dimensionless gravitational acceleration acting in the $y$ direction:

$\rho e=\gamma M^{2}\left(\frac{\rho \boldsymbol{v}^{2}}{2}+\frac{1}{F r} \rho g y\right)+\frac{P}{\gamma-1}$.

Using $P_{\text {ref }} / \rho_{\text {ref }}=T_{\text {ref }} k / \mu$ ( $\mu$ the mean molecular weight, $k$ the Boltzmann constant), the gas pressure results from the dimensionless thermal equation of state of the ideal gas:

$P=\rho T$. 
Complex B:

The dimensionless dust moment equations are $(j \in \mathbb{N})$ :

$\left(\rho L_{0}\right)_{\mathrm{t}}+\nabla \cdot\left(\boldsymbol{v} \rho L_{0}\right)=D a_{\mathrm{d}}^{\mathrm{nuc}} J_{*}$

$\left(\rho L_{j}\right)_{\mathrm{t}}+\nabla \cdot\left(\boldsymbol{v} \rho L_{j}\right)=D a_{\mathrm{d}}^{\mathrm{nuc}} S e_{j} J_{*}+D a_{\mathrm{d}}^{\mathrm{gr}} \frac{j \chi^{\text {net }}}{3} \rho L_{j-1} \cdot($

They are connected to the element conservation equations by the amount of condensed material which depends on the elemental abundance $\epsilon_{x}$ in the gas phase:

$$
\begin{aligned}
\left(\rho \epsilon_{x}\right)_{\mathrm{t}}+\nabla \cdot\left(\boldsymbol{v} \rho \epsilon_{x}\right)= & -\sum_{r=1}^{R}\left(\nu_{r}^{\mathrm{nuc}} E l D a_{\mathrm{d}}^{\mathrm{nuc}} N_{\mathrm{l}} J_{*}\right. \\
& \left.+\nu_{r}^{\mathrm{gr}} E l D a_{\mathrm{d}}^{\mathrm{gr}} \alpha_{r} n_{x, r} v_{\mathrm{rel}, x} \rho L_{2}\right)
\end{aligned}
$$

$\epsilon_{x}$ is the element abundance of the chemical element $x$ (e.g. $\mathrm{Ti}, \mathrm{Si}, \mathrm{O})$ in mass fractions, which is consumed by nucleation (first term, r.h.s.) and growth (second term, r.h.s.) with the stoichiometric factor $\nu_{r}$ due to the reaction $r$. $n_{x, r}$ is the particle density of the atoms and molecules containing the element $x$ which are involved in nucleation and/or growth by reaction $r, L_{j}\left(\left[L_{j}\right]=\mathrm{cm}^{j} / \mathrm{g}\right)$ are the dust moments in mass fractions of the grain size distribution function $f(V)$ with $V$ the grain volume (see Dominik et al. 1993) and are here given for completeness

$\rho L_{j}=\int_{V_{1}}^{\infty} V^{j / 3} f(V) \mathrm{d} V \quad(j=0,1, \ldots)$.

$V_{\mathrm{l}}=N_{\mathrm{l}} V_{0}$ is the lower integration boundary and represents the smallest dust particle considered in the growth process $\left(N_{l}=1000\right)$. $V_{0}$ is the hypothetical monomer volume which is different for different monomer species (e.g. $V_{0, \mathrm{SiO}}=2.926382 \times 10^{-23} \mathrm{~cm}^{3}, V_{0, \mathrm{TiO}_{2}}=3.135233 \times$ $\left.10^{-23} \mathrm{~cm}^{3}\right)$.

The following relations hold (Gail \& Sedlmayr 1988; $\left.\left[\rho L_{j}\right]=\mathrm{cm}^{j} \mathrm{~cm}^{-3}\right)$ :

$\rho L_{0}=n_{\mathrm{d}} \quad$ total number of grains per $\mathrm{cm}^{3}$

$\sqrt[3]{\frac{3}{4 \pi}} \frac{L_{1}}{L_{0}}=\langle a\rangle \quad$ mean particle radius in $\mathrm{cm}$

$\sqrt[3]{36 \pi} \frac{L_{2}}{L_{0}}=\langle A\rangle \quad$ mean dust surface $\mathrm{cm}^{2}$

$\frac{L_{3}}{L_{0}}=\langle V\rangle \quad$ mean dust volume $\mathrm{cm}^{3}$.

The degree of condensation $f_{\mathrm{c}, x}$ is a measure of the number of elements consumed by nucleation and growth of a species containing the chemical element $x$,

$f_{\mathrm{c}, x}=1-\frac{\epsilon_{x}}{\epsilon_{0, x}}$

with $\epsilon_{0, x}$ the initial element abundance of the element $x$.

The system of partial differential equations is closed by various material quantities and equations of state which are partly expressed by non-linear algebraic equations:

- The adiabatic coefficient $\gamma=7 / 5$ for a molecular gas.
- The sticking coefficient $\alpha_{r}$ for a particular reaction $r$ is about 1 (e.g. Rossow 1978; Zöckler 1997).

- The relative velocity $v_{\mathrm{rel}, x}$ of the gas species. It is assumed to be the root mean square of the thermal velocity since the thermodynamic equilibrium velocity is given by a Maxwell distribution,

$v_{\mathrm{rel}, x}=v_{\mathrm{th}, x}=\sqrt{\frac{k T}{2 \pi \bar{\mu}}}$

with $\bar{\mu}=1 /\left(1 / m_{x}+1 / m_{V}\right)$ where $m_{V}$ is the mass of the grain of volume $V$ and $m_{x}$ is the mass of the monomer molecule containing element $x$. Since $m_{V} \gg$ $m_{x}$ for macroscopic grains

$v_{\mathrm{rel}, x} \approx \sqrt{\frac{k T}{2 \pi m_{x}}}$.

- The rate of (homogeneous, homomolecular) nucleation $J_{*}\left[\mathrm{~cm}^{-3} \mathrm{~s}^{-1}\right]$ according to the modified classical nucleation theory derived by Gail et al. (1984) is

$J_{*}=\frac{n_{x}}{\tau} Z \exp \left[\left(N_{*}-1\right) \ln S-\left(\frac{T_{\Theta}}{T}\right) \frac{\left(N_{*}-1\right)}{\left(N_{*}-1\right)^{1 / 3}}\right]$.

The seed growth time scale is $\tau=n_{x} v_{\text {rel }, x} N_{*}^{2 / 3} A_{0}$ for the gaseous nucleation species $x . N_{*}$ is the size of the critical cluster (see Gail et al. 1984), $A_{0}$ is the hypothetical monomer surface and $N_{*}^{2 / 3} A_{0}$ is the surface of the critical cluster. The supersaturation ratio is defined by

$S=\frac{p_{x}}{p_{\text {sat }, x}}$

with $p_{x}=n_{x} k T$ the partial pressure of the nucleation species $x, n_{x}$ the corresponding number density, and $p_{\text {sat }, x}$ the corresponding saturation vapor pressure. $T_{\Theta}=4 \pi a_{0}^{2} \sigma / k$ with $a_{0}$ being the hypothetical monomer radius and $\sigma$ the surface tension (see Eq. (2.7) in Gail et al. 1984). The surface tension for the nucleating clusters are determined from size dependent thermodynamical data (see e.g. Jeong et al. 2000). For $\left(\mathrm{TiO}_{2}\right)_{N}$ a surface tension of $\sigma=800 \mathrm{erg} \mathrm{cm}^{-2}$ is adopted (Jeong 2000, priv. com.). $v_{\text {rel }, x}$ is the thermal velocity between the grain of size $N_{*}$ and gaseous nucleation species, therefore according to Eq. (15):

$v_{\mathrm{th}, x}=\sqrt{\frac{k T}{2 \pi}\left(\frac{1}{m_{x}}+\frac{1}{m_{N_{*}}}\right)}$.

In order to provide an expression for $J_{*}$ which can be handled by asymptotic techniques (see Sect. 3), we adopt the Zeldovich factor $Z=1$ for the studies presented in this paper. We furthermore assume $N_{*} \gg 1$, therefore $N_{*}-1 \approx N_{*}$ (classical nucleation theory). We have tested these simplifications and found that the uncertainties in the saturation vapor pressure have a considerably larger impact on the nucleation rate. According to classical nucleation theory, we adopt

$N_{*}=\left(\frac{2}{3} \frac{T_{\Theta}}{T} \frac{1}{\ln S}\right)^{3}$ 
(compare Eq. (2.16) in Gail et al. 1984) and find the following simplified expression for the nucleation rate:

$$
J_{*}=\frac{n_{x}}{\tau} \exp \left[-\frac{4}{27}\left(\frac{T_{\Theta}}{T}\right)^{3} \frac{1}{\ln S^{2}}\right] .
$$

This expression captures well the key threshold characteristics of the nucleation process (see Sect. 3): efficient nucleation is only possible for temperatures $T_{S}$ with $S\left(T_{S}\right) \gg 1$. It is $T_{S}<T(S=1)$.

- The net growth velocity for heterogeneous dust growth $\chi^{\text {net }}$ is

$$
\chi^{\text {net }}=\sqrt[3]{36 \pi} \sum_{r}^{R} \alpha_{r} n_{r} v_{\mathrm{rel}, r} V_{0, r}
$$

with $V_{0, r}$ the hypothetical monomer volume grown by the reaction $r$. In contrast to the homogeneous nucleation process considered in Eq. (21), more than one growth species is taken into account which causes the summation over $r$ in Eq. (22). In the work presented here we consider only $\mathrm{SiO}$ and $\mathrm{TiO}_{2}$ as growth species such that $\chi^{\text {net }}=\chi^{\mathrm{SiO}}+\chi^{\mathrm{TiO}_{2}}$. The relative velocity $v_{\text {rel, } r}$ can be calculated applying Eq. (16).

- The total absorption coefficient in the energy equation (Eq. (3)) is $\kappa=\kappa^{\text {gas }}+\kappa^{\text {dust }}$ with $\kappa^{\text {dust }} \sim L_{3}$ in the small particle limit of Mie theory $(a \ll \lambda / 2 \pi$, $\lambda$ - photon wave length). A fit to the data for astronomical silicates (Draine \& Lee 1984) yields $\kappa^{\text {dust }}=$ $0.75 L_{3} 1.17 T^{1.12}$ (Gail \& Sedlmayr 1986). The gas opacity per mass is assumed to be constant.

\subsection{Characteristic non-dimensional numbers and scale analysis}

The related estimations of typical time and length scales are summarized in Table 2.1 and have been performed in order to characterize the physical and chemical situation in the atmospheres of brown dwarfs. The choice of the thermodynamical values has been guided by a hydrostatic brown dwarf model atmosphere developed by Tsuji et al. (1999) which is considered as a representative state of the art model of brown dwarf atmospheres ${ }^{2}$.

The dimensionless numbers characterizing our problem are the Mach number $M$, the Froude number Fr, and the Reynolds number Re. Assuming the turbulence velocity to be of the order of the velocity of sound leads to $M \approx 1$, indicating the strong influence of the fluid's compressibility. In fact, in classical 1D stellar atmosphere simulations an additional line broadening component is needed in order to fit observed spectra. This component in generally

\footnotetext{
${ }^{2}$ The model contains the hydrostatic equilibrium consistently coupled to the frequency-dependent radiative transfer equation. A convective flux component is taken into account according to the mixing length theory. The gas phase abundances of atoms, molecules and dust candidates are calculated assuming phase and chemical equilibrium with a prescribed grain size distribution.
}

chosen to be of the order of the velocity of sound (see e.g. Allard et al. 1995; Tsuji et al. 1999) and might be understood as the mean value of a turbulent background velocity field ${ }^{3}$.

The Froude number, which relates the hydrodynamic and the gravitational acceleration, equals one if the density scale height is considered as reference length. If smaller reference lengths $\left(l_{\text {ref }}<H_{\rho}\right)$ are considered, gravitation becomes negligible for the hydrodynamic behavior since $\mathrm{Fr} \gg 1$. Therefore, turbulent mixing and relative velocities between dust and gas (drift) are negligible which is characteristic for microscopic scales. The reference length $l_{\text {ref }}<H_{\rho}$ is chosen much smaller than a typical atmosphere's extension of a few $H_{\rho}\left(H_{\rho} \approx 10^{6} \mathrm{~cm}\right)$. The estimate of the Reynolds number, $R e=10^{6} \ldots 10^{8}$, for a typical brown dwarf atmosphere situation indicates that the viscosity of the gas is too small to damp hydrodynamical perturbations and a turbulent hydrodynamic field can be expected.

In radiatively influenced environments like stellar atmospheres, a characteristic number for the radiative heating / cooling, $R d=4 \kappa_{\text {ref }} \sigma T_{\text {ref }}^{4} \cdot \frac{t_{\text {ref }}}{P_{\text {ref }}}$, can be identified which relates the radiative energy contents and the thermal energy contents of the gas. Depending on typical absorption coefficients, it changes by orders of magnitude, if the absorption coefficient $\kappa$ changes, e.g. due to dust formation (see Table 2.1). Even without dust, $R d$ varies between the inner and the outer layers of the atmosphere because of varying gas absorption coefficients.

The scaling of the dust moment equations provides two dust Damköhler numbers ${ }^{4}$ and a characteristic number for the grain size distribution:

1) $D a^{\text {nuc }}=t_{\text {ref }} J_{*, \text { ref }} / \rho_{\text {ref }} L_{0, \text { ref }}$ is the Damköhler number of dust nucleation and relates the number of newly formed dust particles to the number of dust particles typically formed during one hydrodynamical time $t_{\mathrm{ref}}$;

2) $D a^{\mathrm{gr}}=t_{\mathrm{ref}} \chi_{\mathrm{ref}} /\left(\frac{4 \pi}{3}\langle a\rangle_{\mathrm{ref}}^{3}\right)^{1 / 3}$ is the Damköhler number of dust growth and relates the increase of the grain radius during $t_{\text {ref }}$ by a growth velocity $\chi_{\text {ref }}$ to a typical grain radius $\langle a\rangle_{\text {ref }}$;

3) $S e_{j}=\left(a_{1} /\langle a\rangle_{\mathrm{ref}}\right)^{j}$ is the Sedlmä̈r number which characterizes the dust size distribution and relates the minimum particle size $a_{\mathrm{l}}$ in the definition of the dust moments with a typical grain radius $\langle a\rangle_{\text {ref. It depends on }}$ the order $j(j \in \mathbb{N})$ of the dust moments.

Considering element conservation another characteristic number must be accounted for: $\mathrm{El}=$ $\rho_{\text {ref }} L_{0}^{\text {ref }} N_{\mathrm{l}} / n_{<\mathrm{H}>\text {,ref }} \epsilon_{\text {ref. }}$. It relates the amount of elements consumed by dust grains to a typical elemental abundance $\epsilon_{\text {ref }}$.

\footnotetext{
${ }^{3}$ Gustafsson et al. (1974) discussed the influence of a turbulent pressure component on the hydrostatic atmosphere structure of metal-deficient giant stars.

${ }^{4}$ Damköhler numbers are related to chemical time scales.
} 
Table 1. Characteristic numbers and reference values used for the analysis of microscopic scales. Hydro- and thermodynamic reference values have been guided by static brown dwarf model atmosphere calculations (Tsuji et al. 1999). The reference values for the dust complex have been adopted with regard to the dust formation window. $\left(\gamma=7 / 5, a_{1}=2.145 \times 10^{-7} \mathrm{~cm}, m_{\mathrm{H}}\right.$ mass of the hydrogen atom, $\sigma$ Stefan-Boltzmann constant, $k$ Boltzmann constant, $\mu_{\text {kin }}$ kinematic viscosity, $\left.N_{1}=1000\right)$.

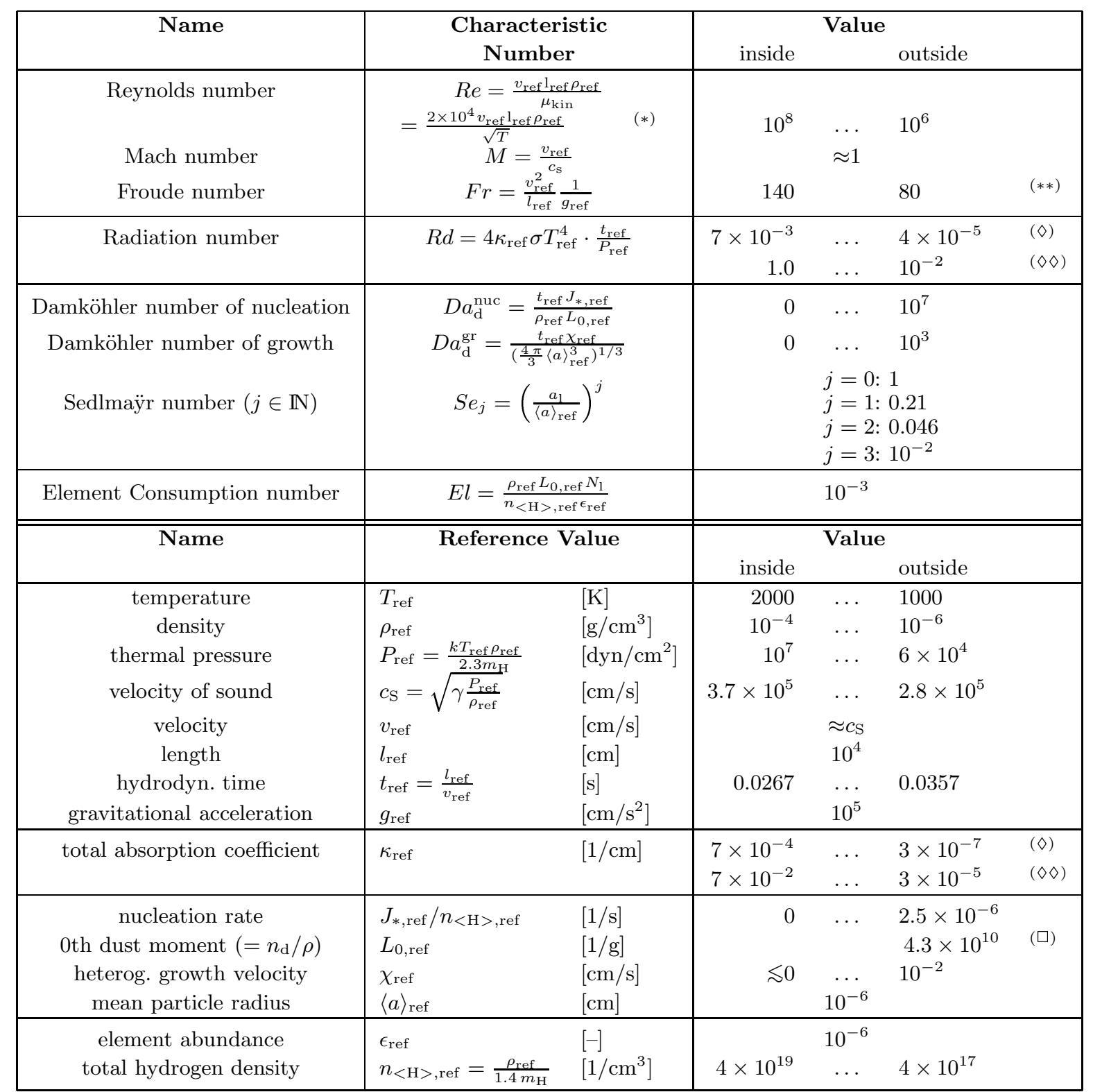

${ }^{(*)}$ For a hydrogen rich gas. ${ }^{(* *)} \mathrm{Fr}=1$ if $l_{\text {ref }}=H_{\rho}, H_{\rho}=$ density scale hight $\left(\approx 10^{6} \mathrm{~cm}\right) .{ }^{(\diamond)}$ gas only, $(\diamond \diamond)$ dust and gas,

() Gail \& Sedlmayr (1999).

The Damköhler numbers of dust nucleation, $D a^{\text {nuc }}$, and dust growth, $D a^{\mathrm{gr}}$, are both large in contrast to the Damköhler number of element consumption, El, inside the dust formation window ( $T \lesssim 1700 \mathrm{~K}$ ). The reason is that usually the nucleation process is much faster than the growth process $\left(\rightarrow\right.$ large $\left.D a^{\text {nuc }}\right)$, and the growth velocity is usually large enough that macroscopic particles $\left(\langle a\rangle \gg\langle a\rangle_{\text {ref }}\right)$ are formed $\left(\rightarrow\right.$ large $\left.D a^{\mathrm{gr}}\right)$. The small $E l$ indicates that only a relatively large amount of dust can significantly affect the elemental abundances which determines the amount of the gaseous species not trapped in dust grains (compare Figs. 3 and 5).
The time scales,

$$
\begin{aligned}
& \tau^{\mathrm{nuc}}=\frac{\rho_{\mathrm{ref}} L_{0, \mathrm{ref}}}{J_{*, \text { ref }}} \approx 10^{-7} \mathrm{~s}, \\
& \tau^{\mathrm{gr}}=\frac{\langle a\rangle_{\mathrm{ref}}}{\chi_{\mathrm{ref}}} \approx 10^{-4} \mathrm{~s},
\end{aligned}
$$

on which dust nucleation (Eq. (23)) and dust growth (Eq. (24)) take place, are much shorter than a typical hydrodynamical time scale $t_{\text {ref }} \approx 10^{-2}$ s well inside the dust formation window $(T \lesssim 1700 \mathrm{~K})$ on the microscopic reference length considered here. The nucleation time scale 
becomes very large $\left(\tau^{\text {nuc }} \approx 1 \mathrm{~s}\right)$ in the regime of inefficient dust formation $(T>1700 \mathrm{~K})$ and is much longer than a typical life time of an acoustic waves on a microscopic scale (see Table 2.1). Therefore, a fast change from the " $\tau^{\text {nuc }}>t_{\text {ref }}$ "-regime to the " $\tau^{\text {nuc }} \ll t_{\text {ref }}$ "-regime occurs if a critical temperature threshold, $T_{\mathrm{S}}$, is crossed and efficient dust formation sets in.

The large difference between $\tau^{\text {nuc }}$ and $\tau^{\text {gr }}$ justifies the separate treatment of seed formation (nucleation) and growth. We thereby confirm the corresponding time scale estimate in Rossow (1978).

The cooling time scale

$\tau^{\mathrm{cool}}=\left(\frac{4 \pi \kappa_{\mathrm{ref}}}{c_{V}} \frac{\partial B}{\partial T}\right)^{-1} \approx 10^{7} \mathrm{~s}$,

with $c_{V}$ the specific heat capacity for constant volume and $B(T)$ the frequency integrated Planck function, is comparably large and the gas will therefore behave adiabatically. The cooling time scale changes strongly if dust has formed, and therefore, a change of the thermodyamical behavior of the gas-dust-mixture from an almost adiabatically to almost instantaneously radiative relaxation can occur. Since dust formation depends very sensitively on temperature, such a changing behavior causes a strong feedback of the hydrodynamics on the dust formation process itself (see Sect. 3).

The analysis of the characteristic numbers shows that the governing equations of our model problem are those of an inviscid, compressible fluid which are coupled to stiff dust moment equations and a singular radiative energy relaxation if dust is present. The dominant interactions in the model Eqs. (1)-(3) occur in the energy equation (Eq. (3)) and in the dust moment equations (Eq. (7)).

\section{An idealized dust formation process}

Our investigations are led by the following model:

In the atmosphere of a brown dwarf, acoustic expansion waves are continuously generated by turbulence. The local temperature might fall below the temperature threshold $T_{\mathrm{S}}$ for effective dust nucleation by the superposition of acoustic expansion waves carrying negative temperature disturbances. During the time of the interaction seed nuclei will form. The temperature increases again after the superposition, and nucleation stops, but the seeds are not destroyed. As the seeds subsequently grow to macroscopic sizes, radiative cooling intensifies, the temperature decreases, and nucleation proceeds further. Thus, dust formation can be initiated by the interaction of acoustic waves in the microscopic scale regime.

This model is used to investigate possible mechanisms to initiate dust formation. It further serves to define a test problem for numerical simulation since analytical, approximate solutions can be obtained by asymptotic techniques.

In the following, we consider an observer co-moving with the local fluid velocity. Such an observer recognizes the interaction of acoustic waves as a fast process leaving local spots of dust nuclei with an initial value for $L_{0}$. The different time scales of the fast acoustic wave interactions and the comparably slow dust growth justifies the following separate treatment of dust nucleation and growth.

We consider a temperature and pressure regime close to effective dust forming conditions. The supersaturation ratio $S=p_{x} / p_{\text {sat }, x}$ can be approximated by introducing material constants $A$ and $B$ for $\ln p_{\text {sat }, x}=A-\frac{B}{T}\left({ }^{5}\right)$ :

$$
\begin{aligned}
S & \approx \frac{n_{x} k T}{\exp \left(A-\frac{B}{T}\right)} \\
& =\hat{S} \exp \left(-B\left(\frac{1}{T_{\mathrm{S}}}-\frac{1}{T}\right)\right)
\end{aligned}
$$

with

$\hat{S}=\frac{n_{x} k T}{p_{\text {sat }, x}^{0} \exp \left(-B / T_{\mathrm{S}}\right)}$,

and $p_{\text {sat }, x}^{0}=e^{A}$ which is constant. The nucleation rate depends very sensitively on $A$ and $B$. In order to enter the dust formation window of efficient nucleation, supersaturation $S \gg 1$ must be achieved. Assuming $\hat{S} \approx 1$ it follows $\ln S \approx B\left(1 / T-1 / T_{\mathrm{S}}\right)$ and, with Eq. (21),

$J_{*}=\frac{n_{x}}{\tau} \exp \left(-\frac{\Theta}{T\left(T_{\mathrm{S}}-T\right)^{2}}\right), \quad \Theta=\frac{4}{27} \frac{1}{B^{2}} T_{\mathrm{S}}^{2} T_{\Theta}^{3}$.

A key observation for the analysis is that the nucleation rate $J_{*}$ strongly depends on temperature with a reverse activation energy type characteristic. The number of dust nuclei formed in a time interval $\Delta t$ is given by the time integral $n_{\mathrm{d}}=\rho L_{0}=\int_{\Delta t} J_{*}(T(t)) \mathrm{d} t$, where $\Delta t$ is the time of interaction between two waves (Fig. 1, l.h.s.). Under typical dust forming conditions the parameter $\Theta$ in Eq. (29) is very large, here $\Theta=\mathcal{O}\left(10^{9}\right)$. This allows an asymptotic approximation of the integral in the form

$$
\begin{aligned}
\int_{t} J_{*} \mathrm{~d} t & =\int f(t) \exp (\Theta g(t)) \mathrm{d} t \\
& \approx \frac{\sqrt{2 \pi} f\left(t_{*}\right)}{\sqrt{-\Theta g^{\prime \prime}\left(t_{*}\right)}} \exp \left(\Theta g\left(t_{*}\right)\right)
\end{aligned}
$$

where $f(t)=n_{x} / \tau(T(t))$ and $g(t)=-\frac{1}{T\left(T_{s}-T\right)^{2}}$ (Bender $\&$ Orzag 1999). Here, $t_{*}$ is the time of the minimum temperature during the interaction. Assuming linear acoustic waves (e.g. Gaussian profile) with given temperature disturbances $\Delta T$, we can analytically evaluate the integral. Figure 1, r.h.s., shows the excellent agreement between a numerical computation of the integral and the asymptotic approximation (Eq. (30)) for different temperature amplitudes.

To study the effects of multiple stochastic interactions of this type, we consider 86 Gaussian-shaped acoustic waves with random amplitudes $\Delta T[\mathrm{~K}] \in[-100,100]$,

\footnotetext{
${ }^{5}$ For example, the saturation vapor pressure $\ln p_{\mathrm{sat}, \mathrm{TiO}_{2}}=$ $35.8027-74734.7 / T$ for $\mathrm{TiO}_{2}$ has been fitted from the electronic version of the JANAF tables 1985 (Chase et al. 1985) for the temperature interval $500 \mathrm{~K} \ldots 2500 \mathrm{~K}$.
} 

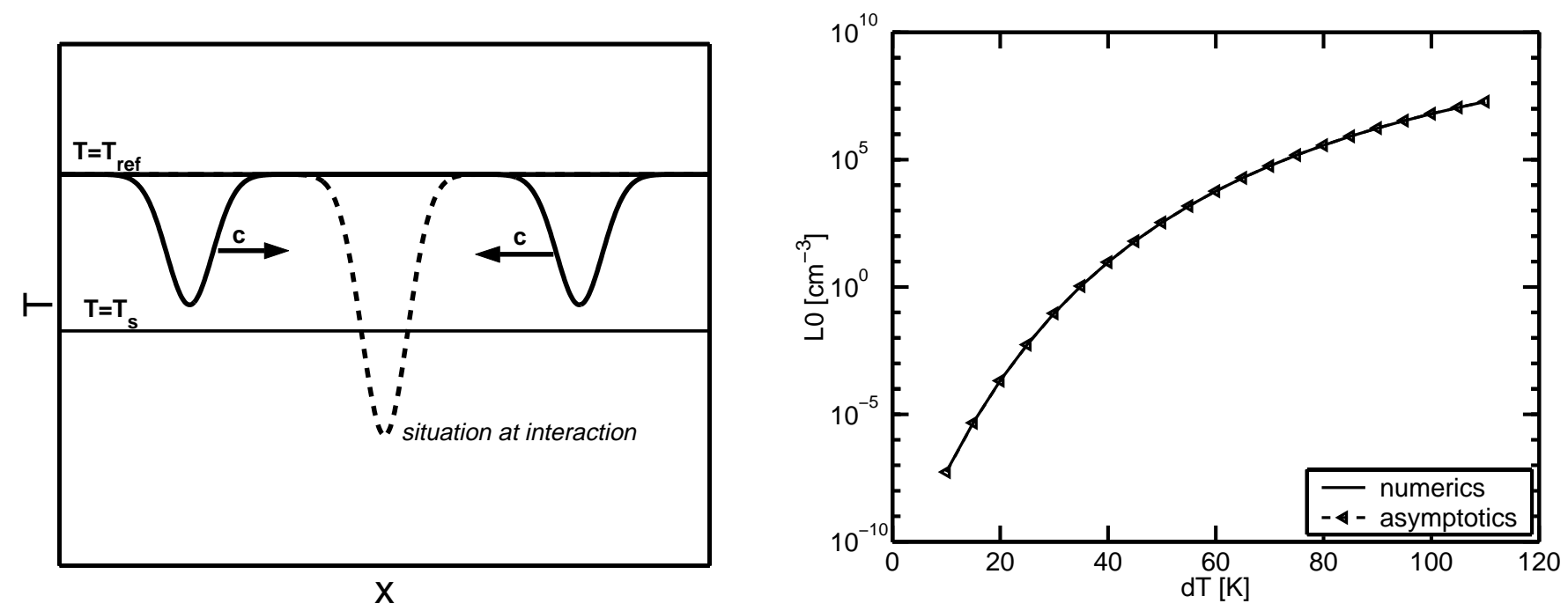

Fig. 1. Left: two interacting waves with identical wavelength and amplitudes. Right: evaluation of the seed formation integral $L_{0}=\int_{\Delta t} J_{*}(T(t)) \mathrm{d} t$ by numerical integration and asymptotic evaluation (Eq. (30)).
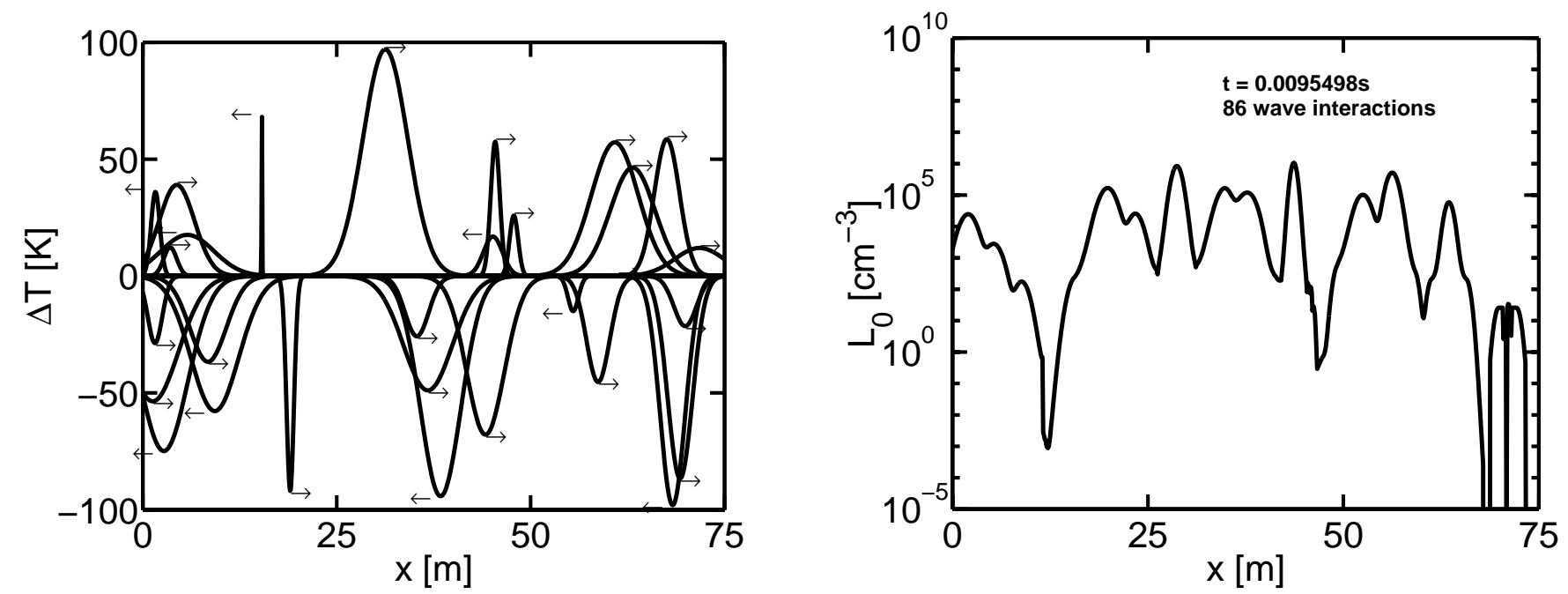

Fig. 2. Left: stochastic interaction of 86 Gaussian acoustic waves. Right: resulting spatial distribution of dust nuclei of 86 Gaussian acoustic waves.

wavelengths $\lambda[\mathrm{cm}] \in[0,500]$, and propagation directions (Fig. 2, l.h.s.). Since every wave travels with the same speed of sound here, only waves moving in opposite directions interact. If two waves with a negative temperature fluctuation collide, seed formation occurs during the time of interaction. Figure 2 (r.h.s.) displays the spatial distribution of dust nuclei, represented by the zeroeth dust moment $L_{0}$ (Eq. (10)), after the interaction of all the acoustic waves. For the computation of the spatial nuclei distribution we used the asymptotic Eq. (30) assuming linear superposition of the waves.

Even with short interaction times of the order $t=$ $\lambda / c=\mathcal{O}\left(10^{-3} \mathrm{~s}\right)$, we see locally $\mathcal{O}\left(10^{2}\right)$ dust nuclei per unit volume in Fig. 2. Comparing the time scales of seed nucleation by interaction of turbulence generated acoustic waves and the time scales for a complete dust growth process in Fig. 3, the separate investigation of the nucleation process alone here is fully justified.
For the long term evolution of a complete grain growth process we investigate the time evolution of a single gas cell in Lagrangian space after the initiation of the dust growth cycle by stochastic acoustic waves. This corresponds to following a gas cell along particle trajectories with initial conditions on $L_{0}$ given by one point in Fig. 2. For an observer moving with the gas cell, the flow is governed to leading order by the equations for low Mach numbers. Assuming a constant background pressure, the energy equation in Lagrangian space can be reduced to the the following equation:

$$
\frac{\mathrm{d} T}{\mathrm{~d} t}=R d \kappa\left(1-T^{4}\right) T
$$

The density is then given by the equation of state. Together with the dust moment Eqs. (6), (7) and the element consumption Eq. (8) we have a closed set of 
equations describing the dust formation process observed by a co-moving observer.

The long term evolution of dust formation has been set up as a test problem in order to validate the code solving the full Eulerian model problem (Eqs. (1)-(8)) against the presented simplified model. The results will be discussed in comparison to the solution of the full model problem in Sect. 4.2 .

\section{Numerical simulations}

\subsection{Approach}

The fully time-dependent solution of the model equations has been obtained by applying a multi-dimensional Euler solver for compressible fluids which has been extended in order to treat the complex of dust formation (Eqs. (6)-(7)) and elemental conservation (Eq. (8)) consistently coupled to the hydro- and thermodynamics (Eqs. (1)-(4)). The hydro-/thermodynamics code has already been the subject of several studies in computational science (Klein 1995; Smiljanovski et al. 1997; Schneider et al. 1999).

Numerical Features: The hydro code is a second-order Godunov-type compressible Euler solver (Smiljanovski et al. 1997) for a multi-dimensional Cartesian grid. The operator splitting method is applied for direction, dust formation, chemistry, radiative cooling, and gravity. Due to the smaller time scales of the dust complex, the dust moment and element conservation equation are solved applying the CVODE solver (Cohen \& Hindmarsh 2000; LLNL) in the framework of the operator splitting method.

Assumptions: The gas is considered as an ideal gas with constant molecular weight $\mu=2.3$ and adiabatic index $\gamma=7 / 5$. The dust grains are core-mantle grains with a homogeneous core of $\left(\mathrm{TiO}_{2}\right)_{N}$ (Gail \& Sedlmayr 1998; Jeong 2000) and a heterogeneous mantel composed of $\mathrm{SiO}$ and $\mathrm{TiO}_{2}$. The thermodynamic data for the $\left(\mathrm{TiO}_{2}\right)_{N}$ cluster have been obtained from density functional quantum mechanical model calculations by Jeong et al. (2000). The dust opacity is treated in the small particle limit of Mie theory. Three elements ( $\mathrm{Si}, \mathrm{Ti}, \mathrm{O}$ ) are involved in the dust formation, therefore, three equations of Eq. (8) have to be solved.

In the following example calculation we assume that $\mathrm{TiO}_{2}$ and $\mathrm{SiO}$ are the most abundant molecules which contain $\mathrm{Ti}$ and $\mathrm{Si}$, respectively. According to the equilibrium chemistry used (based on the electronic version of JANAF tables 1985), this assumption is well justified for $\mathrm{SiO}$ in the $(T, \rho)$ range considered here. The situation is not as clear for $\mathrm{TiO}_{2}$ but it is the most abundant Ti-bearing molecule for the following situations considered. Taking advantage of this, we adopted a simple algebraic equation, $n_{x}=\epsilon_{x} n_{<\mathrm{H}>}$, for calculating the number density of $\mathrm{TiO}_{2}$ and $\mathrm{SiO}$ in order to be able to obtain some long-term behavior of the dust complex. Thereby, we can abandon

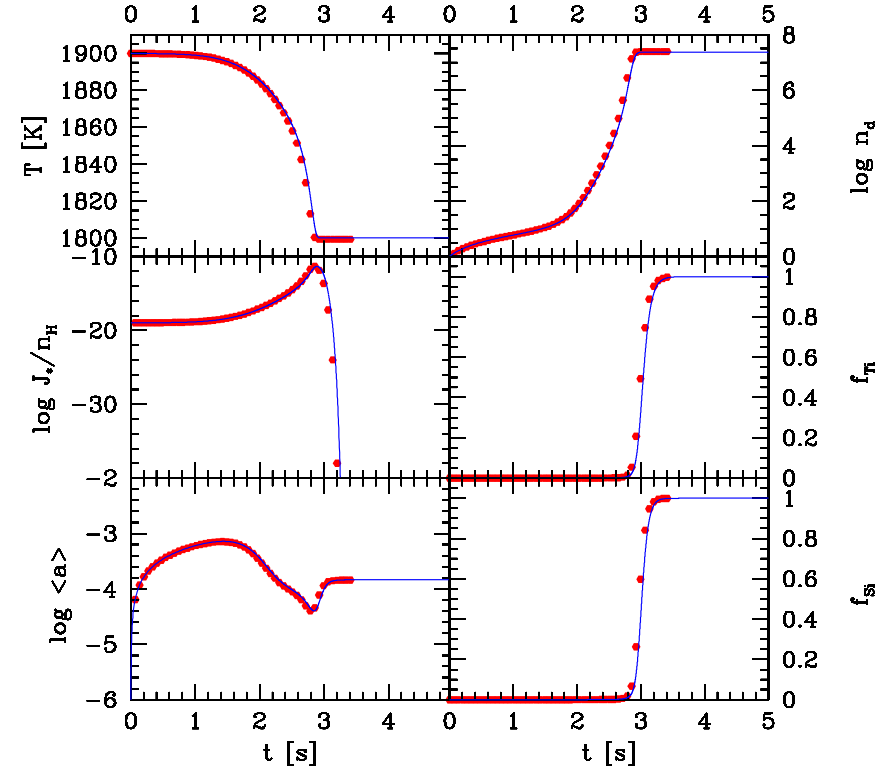

Fig. 3. Long term behavior of the dust formation process according to a result of asymptotic techniques (asterisks; a)) and numerical simulation of the full model problem (solid line; b)). The calculations considere the nucleation of $\left(\mathrm{TiO}_{2}\right)_{N}$ clusters and their growth by $\mathrm{TiO}_{2}$ and $\mathrm{SiO}$ addition. Initial conditions: $T(x, 0)=1900 \mathrm{~K}\left(T_{\mathrm{RE}}=1800 \mathrm{~K}\right), \rho(x, 0) L_{0}(x, 0)=1 \mathrm{~cm}^{-3}$ and $L_{1}(x, 0) \ldots L_{3}(x, 0)=0$. The density is kept constant.

the chemical equilibrium calculation and save half of the computing time.

\subsection{Validation of the full model code}

Figure 3 compares the long term evolution of the grain growth process computed with

a) a simplified zero dimensional incompressible flow model where a single Lagrangian gas cell after the initiation of nucleation by stochastic wave interaction is considered in the incompressible limit (asterisks, Fig. 3; see also Sect. 3), and

b) the numerical simulation of the full set of equations (Eqs. (1)-(8); solid line, Fig. 3).

The density is kept constant in this test calculation. Both calculations consider $\left(\mathrm{TiO}_{2}\right)_{N}$ nuclei and $\mathrm{SiO} / \mathrm{TiO}_{2}$ growth. As radiative equilibrium temperature, $T_{\mathrm{RE}}=$ $1800 \mathrm{~K}$ is considered which is well below the critical temperature for dust nucleation. A corresponding radiation field is thereby assumed to be present.

Initial values: The initial gas temperature, $T(x, 0)=$ $1900 \mathrm{~K}\left(=T_{\text {ref }}\right)$, is above the critical temperature $T_{\mathrm{S}}$ for efficient nucleation. For the dust moments we set $\rho(x, 0) L_{0}(x, 0)=1 \mathrm{~cm}^{-3}$ and zero for the higher moments. The value for $\rho(x, 0) L_{0}(x, 0)=1 \mathrm{~cm}^{-3}$ can, for example, easily be reached through the interaction of acoustic waves in such a way that the local temperature falls below the critical temperature for dust nucleation for a short time period. 
Results: Both codes yield similar results for the test problem (Fig. 3):

In the beginning, up to about $1 \mathrm{~s}$, the temperature decreases only very little. The radiative cooling is very inefficient due to the small opacity of the gas and the dust. Meanwhile, the initially present small number of dust grains has grown to a mean size of $\approx 10^{-3} \mathrm{~cm}$ and the particle number density $n_{\mathrm{d}}$ has only slightly increased due to the very low rate of nucleation $J_{*}$. At some point, the dust particles are large enough to intensify the radiative cooling. The temperature becomes low enough that the threshold of effective nucleation is again crossed such that the rate of nucleation increases rapidly by about 8 orders of magnitude. Consequently, the number of dust particles increases to its final value. This value becomes constant in time if nucleation stops because all the available material has been consumed. The total consumption occurs in a very small time interval for both, $\mathrm{Si}$ and $\mathrm{Ti}$, which can be seen from the degree of condensation $f_{\mathrm{Si}}=1$ and $f_{\mathrm{Ti}}=1$ (Eq. (14)). At the same time, also the mean particle radius has reached a constant size after it passed a minimum which corresponds to the time of maximum $J_{*}$.

At the same time when new dust particles have been formed, the radiative cooling has again intensified (steeper temperature slope) and the radiative equilibrium temperature has been reached. It should, however, be noticed that the time scales considered are very long in the framework of microscopic scales. A typical reference time is the time for an acoustic waves to cross a path of $1 / 10$ of the density scale hight, which is $\sim 0.03 \mathrm{~s}$ (compare Table 2.1 ). The results can be summarized as follows:

$t \lesssim 1$ s: initially present grains (seed particles) grow;

$1 \mathrm{~s} \lesssim t \lesssim 2 \mathrm{~s}$ : temperature low enough to initiate further nucleation;

$2 \mathrm{~s} \lesssim t \lesssim 2.8 \mathrm{~s}$ : radiative cooling intensifies due to dust formation;

$t \sim 2.8 \mathrm{~s}$ : gaseous $\mathrm{Ti}$ and $\mathrm{Si}$ are consumed and nucleation and growth stops radiative equilibrium is reached.

The long-term behavior of the dust component has been simultaneously described by the full Eulerian description of the multi-dimensional code and the Lagrangian description of a simplified model. The agreement of the results gives us confidence in the successful coupling between the full fluid dynamics (Eqs. (1)-(4)) and the dust complex (Eqs. (6)-(8)) carried out in the dimensionless formulation. We proceed therefore to study the interaction between turbulent motion and dust formation in the framework of more complex scenarios in $1 \mathrm{D}$ and in $2 \mathrm{D}$ on microscopic scales.

\subsection{Intermittent distribution of dust}

The initiation of dust formation in hot atmospheric regions needs a cooling scenario in order to provide appropriately low temperatures to enter the dust formation

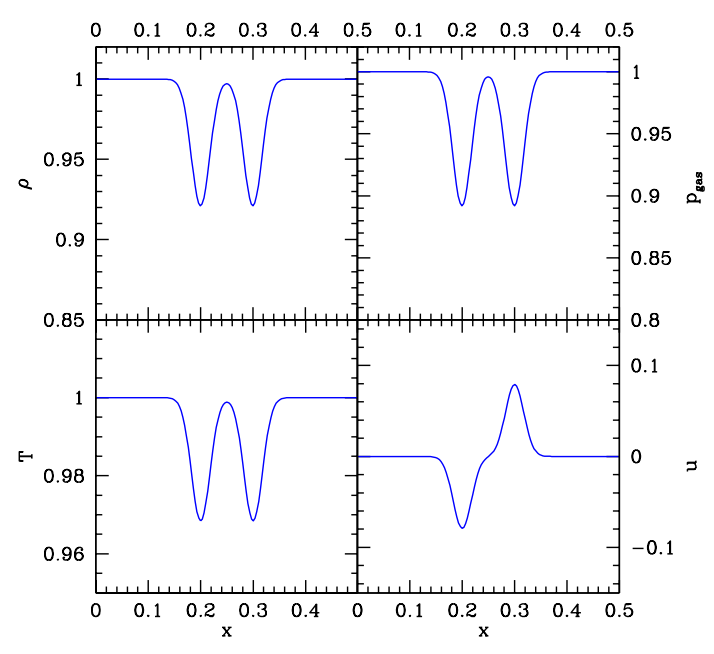

Fig. 4. Initial condition for one-dimensional acoustic waves creating temperature disturbances of $\Delta T=-60 \mathrm{~K}$ with a wavelength of $\lambda=250 \mathrm{~cm}$ moving in a dust-free $\left(L_{0}(x, 0) \ldots L_{3}(x, 0)=0\right)$ gas. The situation investigated $\left(T_{\text {ref }}=1900 \mathrm{~K}, \rho_{\text {ref }}=10^{-4} \mathrm{~g} \mathrm{~cm}^{-3},|\boldsymbol{v}|_{\text {ref }}=3.1 \times 10^{5} \mathrm{~cm} \mathrm{~s}^{-1}\right.$, $t_{\text {ref }}=0.032 \mathrm{~s}$ ) simulates the dust formation under turbulent conditions in a relatively hot and dense region of a brown dwarf atmosphere.

window. Intense convective motion creates interacting acoustic waves which may cause local temperature decreases low enough to initiate dust nucleation. A first study of this scenario in a zero-dimensional approach has been used as test problem for the full model code in the previous section.

In the following, the influence of interacting acoustic waves on the dust formation shall be studied in 1D and in $2 \mathrm{D}$. The understanding of the one-dimensional simulations (Sect. 4.3.1) is seen as necessary for the understanding of the more complex multi-dimensional simulations (Sect. 4.3.2). The 2D simulation will provide insight into the spatial distribution of the dust component as a result of multiple interacting acoustic waves.

The following two scenarios have been set up:

(1) 1D expansion wave interactions enforce effective dust formation in an otherwise dust-hostile environment;

(2) 2D random pressure pulses create inhomogeneous distribution of dust.

The reference values (see Table 2.1) are chosen such that the hot and dense region of the brown dwarf atmosphere, hostile to dust formation, is considered.

\subsubsection{Scenario (1): $1 \mathrm{D}$ expansion wave interaction}

The first scenario, where we study an one-dimensional setup, investigates the interaction of acoustic expansion waves which cause a disturbance of the initially homogeneous temperature and density field.

Initial conditions: The initially homogeneous gas temperature $T(x, 0)=1900 \mathrm{~K}\left(=T_{\text {ref }}\right)$ is above the critical temperature $T_{\mathrm{S}}$ for efficient nucleation. Two one-dimensional 


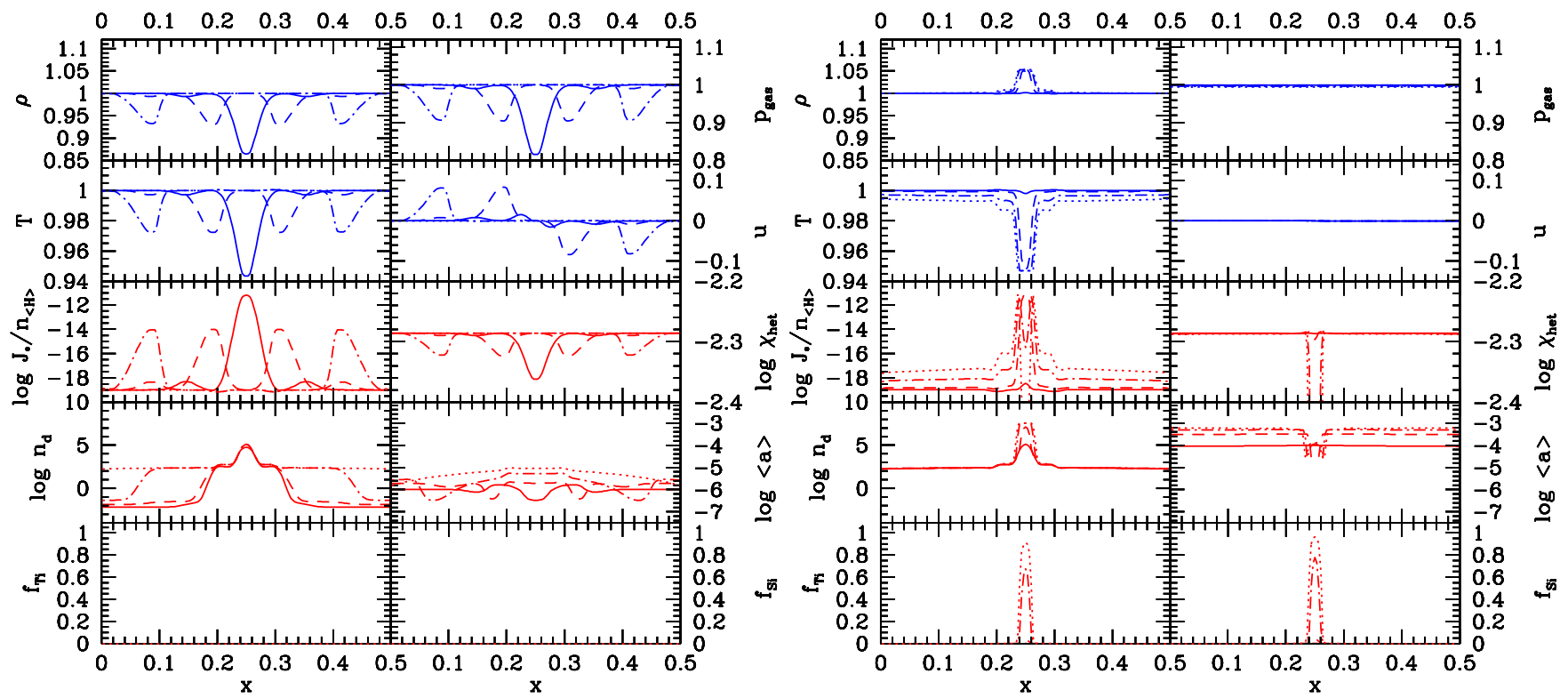

Fig. 5. Left: four snapshots of two interacting acoustic expansion waves and their influence on the dust complex (solid $=$ $1.46 \times 10^{-3} \mathrm{~s}$, dashed $=3.12 \times 10^{-3} \mathrm{~s}$, dash-dot $=6.22 \times 10^{-3} \mathrm{~s}$, dotted $=0.01 \mathrm{~s}$ ). The last instant of time on the l.h.s. (dotted) depicts the quasi-equilibrium state whereafter the system is only determined by the dust growth process until radiative cooling sets in (r.h.s.). Right: four later snapshots where efficient radiative cooling sets in due to the dust formed by two interacting acoustic waves $($ solid $=0.09 \mathrm{~s}$, dashed $=0.3 \mathrm{~s}$, dash-dot $=0.48 \mathrm{~s}$, dotted $=0.58 \mathrm{~s})$. (First row: $\rho$ gas density, $P_{\text {gas }}$ gas pressure, second row: $T$ temperature, $u$ hydrodynamic velocity in $x$-direction, third row: $\log J_{*} / n_{<\mathrm{H}>}\left[\mathrm{s}^{-1}\right]$ nucleation rate per hydrogen atom, $\log \chi^{\text {net }}\left[\mathrm{cm} \mathrm{s}^{-1}\right]$ net growth velocity, fourth row: $\log n_{\mathrm{d}}$ number density of dust particles $\left[\mathrm{cm}^{-3}\right], \log \langle a\rangle$ mean particle radius $[\mathrm{cm}]$, fifth row: $f_{\mathrm{Ti}}, f_{\mathrm{Si}}$ degree of condensation for $\mathrm{Ti}$ and $\mathrm{Si}$ ).

Gaussian-shaped isentropic pressure pulses initiate temperature disturbances of $\Delta T=-60 \mathrm{~K}$ of an arbitrary wavelength of $\lambda=250 \mathrm{~cm}$ (Fig. 4). The gas is initially dust free. The boundaries are nearly transparent, homogeneous von Neumann boundary conditions and a spatial resolution of 150 grid points have been used. The size of the domain is $l_{\mathrm{ref}} / 2=0.5 \times 10^{4} \mathrm{~cm}$.

Results I: Superposition of acoustic expansion waves. Figure 5 (l.h.s.) depicts the time evolution of two interacting acoustic waves and their influence on dust formation until a stationary situation is reached. Stationarity is reached after $\sim 0.01 \mathrm{~s}$ (dotted line) when the sound waves have propagated through the 1D test volume, characterized by a homogeneous temperature, density, gas pressure distribution and a zero hydrodynamic velocity. During the time interval depicted on the 1.h.s. of Fig. 5, the interacting sound waves cause the appearance of an inhomogeneous distribution of dust observable from the dust particle number density $n_{\mathrm{d}}$ which is preserved as long as the stationary state of $T, \rho, P_{\text {gas }}$, and $u$ is conceived.

The initial pressure pulses are already large enough to reach a temperature where dust nucleation is possible but on a very low rate (solid line, l.h.s.), i.e. still outside the window of efficient dust formation. At the time when $\left(t=1.46 \times 10^{-3} \mathrm{~s}\right)$ and the site where $(x=0.25)$ the acoustic expansion waves constructively interfere, the temperature decreases considerably (solid line). This causes the nucleation rate per hydrogen atom $J_{*} / n_{<\mathrm{H}>}$ to increase by 7 orders of magnitude due to its high temperature sensitivity. The dust particle number $n_{\mathrm{d}}$ increases accordingly. Meanwhile, the mean grain size $\langle a\rangle$ has slightly increased but is smaller where the dust nucleation has efficiently taken place during the wave interaction.

As the propagating sound wave solution suggests, the waves penetrate each other and continue to travel in their initial direction. Ideally, they should maintain their original amplitudes. Since the temperature disturbances are still large enough for some dust nucleation (compare $J_{*} / n_{<\mathrm{H}>}$ in Fig. 5, l.h.s.), the dust particle number distribution broadens its shoulders which do, however, not reach the high value of the constructive interference event at $t=1.46 \times 10^{-3} \mathrm{~s}$ and $x=0.25$ (solid line). While the waves continue to pass the 1D test volume, the growth process continues, too. Again, at sites and times of highest $J_{*}$, the mean particle radius is smallest because the growth material is distributed over more and more particles.

As soon as the disturbances have left the domain, temperature, density, and gas pressure become homogeneous and $u=0$. Consequently, the temperature is again too high to allow dust nucleation, therefore $J_{*} \approx 0$ and no further increase of the dust particle number density occurs. But the growth velocity is non-zero, therefore, the dust complex is now governed by a steady increase of the dust particle sizes by the grow of the dust mantel until radiative cooling becomes efficient enough. 


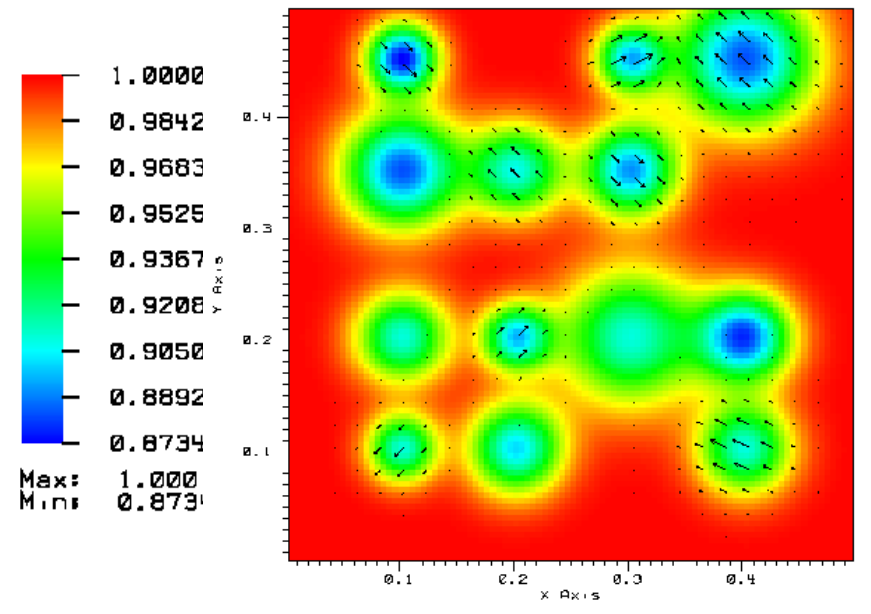

Fig. 6. Initial pressure and velocity field $\left(|\boldsymbol{v}|_{\max }=4.96 \times\right.$ $\left.10^{5} \mathrm{~cm} \mathrm{~s}^{-1},|\boldsymbol{v}|_{\min }=0\right)$. The ordinate indicates the $x$-direction, the abscissa the $z$-direction (= equi-gravitational plane). The pressure pulses initiate random temperature disturbances between $50 \mathrm{~K}$ and $70 \mathrm{~K}$ with random wavelength between $300 \mathrm{~cm}$ and $600 \mathrm{~cm}$. The situation studied is comparable to Fig. 4 .

Results II: Re-initiation of dust formation. Radiative cooling by dust sets in after the grains have grown to a mean size of about $10^{-4} \mathrm{~cm}(=1 \mu \mathrm{m})$ in the calculation (Fig. 5). While the gas pressure stays constant, the temperature decrease causes a corresponding increase of the density which will support the dust formation process, too. Now, the same run-away effect sets in as outlined in our model in Sect. 4.2: the more dust particles are formed the faster the temperature decreases by radiative cooling. This in turn causes more dust to form. The gas temperature has reached the radiative equilibrium temperature and the run-away is stopped (Fig. 5, r.h.s., dashed line).

As time proceeds, the available material is consumed by dust nucleation and growth and the degrees of condensation of $\mathrm{Ti}$ and $\mathrm{Si}, f_{\mathrm{Ti}}$ and $f_{\mathrm{Si}}$, approach unity. As $f_{\mathrm{Ti}}$ increases, the nucleation rate decreases (compare dashed and dash-doted line, r.h.s, Fig. 5) due to less and less material being available to form seed nuclei. Consequently, the growth by $\mathrm{SiO}$ is more efficient after $\approx 0.5 \mathrm{~s}$ inside the wave interaction zone and the mean particle size increases. The abundance of Si decreases faster, i.e. $f_{\mathrm{Si}}$ approaches 1 faster than $f_{\mathrm{Ti}}$ (dash-dot, r.h.s, Fig. 5). After the dust formation is completed in the wave interaction zone $(x \sim 0.25), S_{\mathrm{SiO}}=S_{\mathrm{TiO}_{2}}=1$. Nevertheless, $\mathrm{TiO}_{2}$ and $\mathrm{SiO}$ are still present in the gas phase but only with a partial pressure equal to the saturation vapor pressure.

Meanwhile, also the temperature of the shoulders has decreased and nucleation has been re-initiated. Again, a run-away effect drives the temperature towards $T_{\mathrm{RE}}$ and the dust formation stops if $S_{\mathrm{SiO}}=S_{\mathrm{TiO}_{2}}=1$ is reached.

\subsubsection{Scenario (2): Random, 2-dimensional acoustic pressure pulses}

It has been shown in the last section that acoustic expansion waves may allow the initiation of efficient dust nucleation for a short time. This model scenario shall now be studied in the framework of two-dimensional simulations of the full model problem. The initial values are set up such that a time-evolution of $2 \mathrm{D}$ isentropic, acoustic waves and their influence on the dust formation in the microscopic scale regime are considered.

Initial conditions: The initial gas temperature $T(x, 0)=$ $1900 \mathrm{~K}\left(=T_{\text {ref }}\right)$ is above the critical temperature $T_{\mathrm{S}}$ for nucleation. Two-dimensional Gaussian pressure pulses create an inhomogeneous distribution of pulses of various wavelengths $(\lambda=300 \ldots 600 \mathrm{~cm})$ and temperature amplitudes $(\Delta T=50 \ldots 70 \mathrm{~K})$. The simulations are carried out with a spatial resolution of $100 \times 100$ points. The open boundaries are represented by homogeneous von Neumann boundary conditions. The size of the domain is $l_{\text {ref }} / 2=0.5 \times 10^{4} \mathrm{~cm}$.

Results: Figures 7-9 depict the time evolution of the temperature, density, and velocity distributions (Fig. 7), the nucleation rate (Fig. 8), the dust particle number density and mean particle radius (Fig. 9) as results of interaction of various acoustic waves moving randomly in a $2 \mathrm{D}$ test volume. The instants of time illustrate the system's evolution until a quasi-stationary state is reached (compare Sect. 4.3.1).

The temperature and density distributions in the $2 \mathrm{D}$ test volume seem very similar during the whole time interval depicted. Note, that both values and the velocity are plotted dimensionless and the different scales are plotted next to the graphs and are given in the caption, respectively. As it is characteristic for acoustic waves, an increase of density corresponds to an increase in temperature which can be observed in the colliding (red) zones at $(x, y) \approx(0.1,0.35)$ or $(x, y) \approx(0.4,0.45)$ in Fig. 7 , middle panels. The net direction of the velocity vector indicates the colliding course of the gas at these sites (Fig. 7, the velocity vector field is superimposed to the scalar density field) and it directly results in an inhibition of dust nucleation (Fig. 8, middle panel, blue regions). The particle number density and the mean grain radius are not much affected since the temperature increases to such moderate values that evaporation does not set in (Fig. 9, top and middle panels). However, such interruptions of dust nucleation due to local temperature increases will support the formation of an inhomogeneously distributed dust component on a longer time scale.

In the middle panel (l.h.s.) of Fig. 7 deep blue/black $((x, y) \approx(0.25,0.4),(0.15,0.15))$ regions can be identified which at that time of the simulation have low temperatures due to the constructively interfering expansion waves. The comparison with earlier instants of time (top panels) supports this since no pressure pulses have been initially present at these sites. This pattern of temperature minima is directly mapped by the nucleation rate (Fig. 8, middle panel): $J_{*}$ is highest where the temperature is lowest $((x, y) \approx(0.25,0.4),(0.15,0.15))$ indicating 

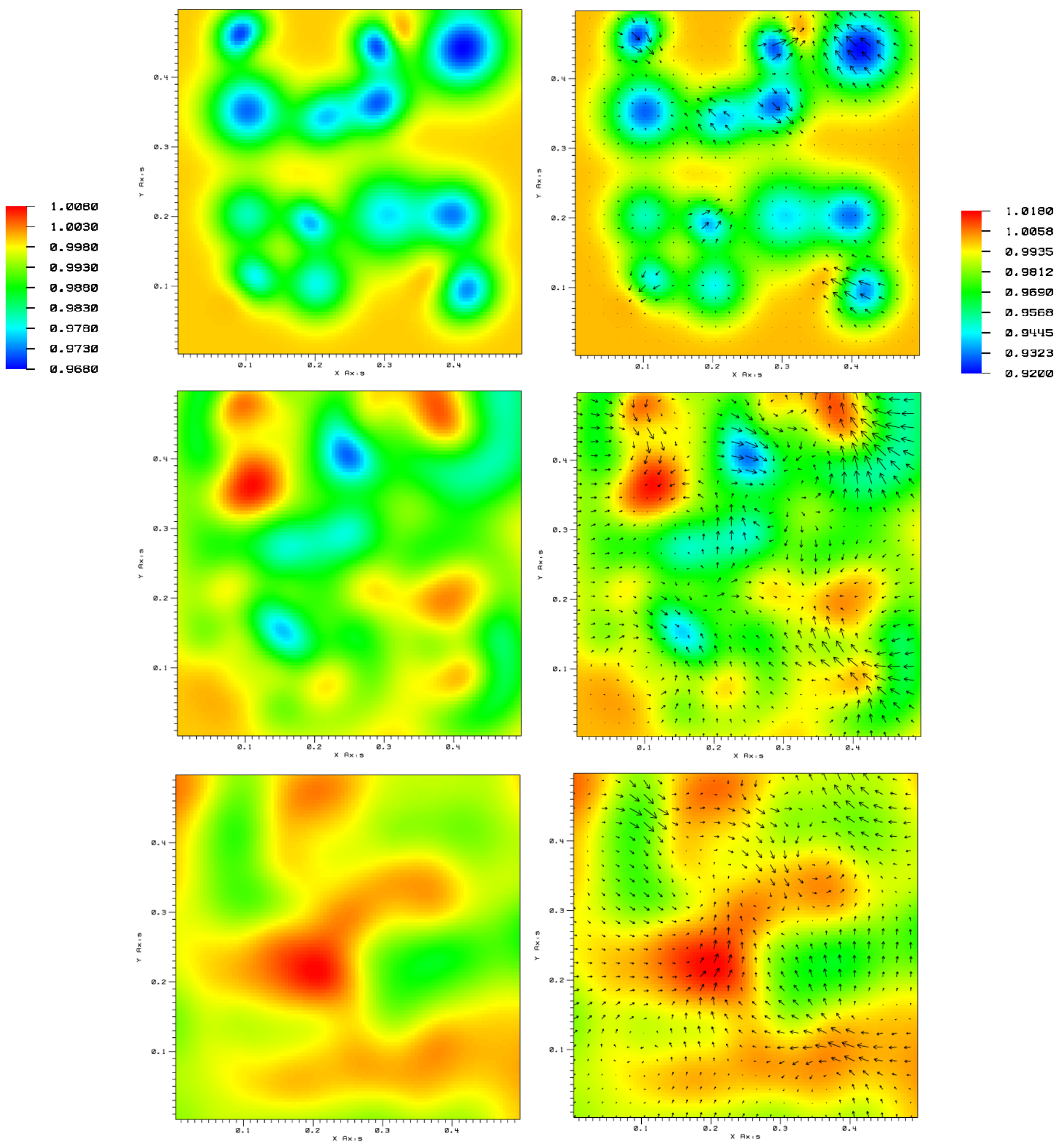

Fig. 7. Time evolution (top to bottom) of the temperature (left) and the density (right). The scaler density field superimposed is the velocity vector field. The interval of temperature and density plotted is given beside the plots left and right, respectively. The values are plotted dimensionless. (Top: $t=3.2 \times 10^{-4} \mathrm{~s},|\boldsymbol{v}|_{\max }=4.03 \times 10^{4} \mathrm{~cm} \mathrm{~s}^{-1},|\boldsymbol{v}|_{\min }=0.077 \mathrm{~cm} \mathrm{~s}^{-1}$; middle: $t=3.3 \times 10^{-3} \mathrm{~s},|\boldsymbol{v}|_{\max }=2.5 \times 10^{4} \mathrm{~cm} \mathrm{~s}^{-1},|\boldsymbol{v}|_{\min }=30.4 \mathrm{~cm} \mathrm{~s}^{-1} ;$ bottom: $t=5 \times 10^{-3} \mathrm{~s},|\boldsymbol{v}|_{\max }=2.4 \times 10^{4} \mathrm{~cm} \mathrm{~s}^{-1}$, $|\boldsymbol{v}|_{\min }=34.1 \mathrm{~cm} \mathrm{~s}^{-1}$.)

an efficient dust formation process. During these events of efficient nucleation, the mean particle radius decreases while the dust number density increases (Fig. 9, middle panels). 

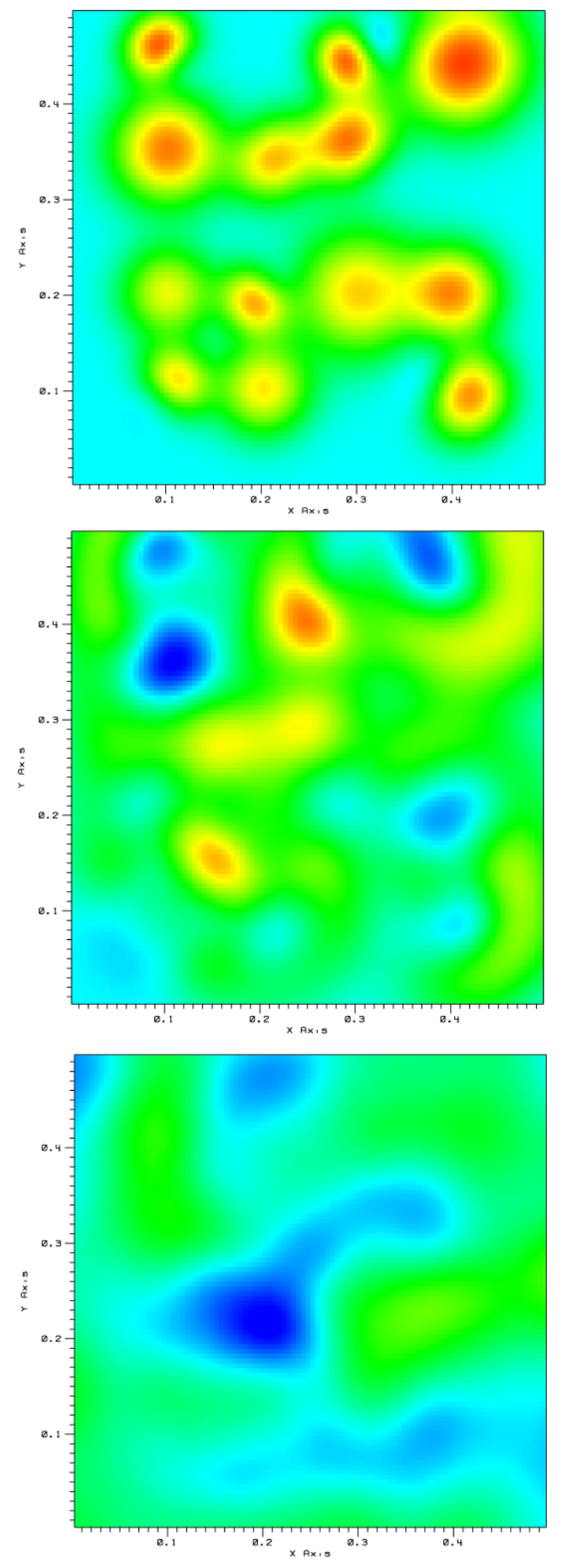

Fig. 8. Time evolution (top to bottom) of the nucleation rate $\log J_{*} / n_{<\mathrm{H}>}\left[\mathrm{s}^{-1}\right]$. The color code is given left beside the plots. The instants of time depicted are the same like in Fig. 7. (Top: $t=3.2 \times 10^{-4} \mathrm{~s}$, middle: $t=3.3 \times 10^{-3} \mathrm{~s}$, bottom: $t=$ $5 \times 10^{-3}$ s.)

As time proceeds, the temperature minima are gone and instead, large regions of temperatures too high for dust formation appear. Consequently, the nucleation rate drops and reaches its minimum (Figs. 7 and 8, last panels).

From now on, the hydro-/thermodynamic quantities return to their (initial) equilibrium levels until radiative cooling becomes effective. The dust complex is governed only by continuous growth of the existing particles. This can be observed from the evolution of the mean particle radius in the two last time steps depicted in Fig. 9 (r.h.s., middle and bottom panels). Here, an increase of the mean particle size occurs in the whole test volume uniformly (compare color changes).

Summarizing we can say that we detected two effects from the 2D simulation which cause inhomogeneous dust formation and distribution:

- randomly interacting acoustic expansion waves create thermodynamic conditions (i.e. low temperatures) in regions usually unfavorable for dust formation;

- corresponding compression waves suppress dust formation in regions appropriate for dust formation due to an increase of temperature.

Both effects are active on very short time scales when the medium is disturbed by acoustic waves. It results in an inhomogeneous distribution of dust per mass with a relatively homogeneous mean particle size. The hydro- and thermodynamic values relax to their equilibrium level until radiative cooling sets in effectively.

\section{Discussion}

The results presented in the last sections have been outlined utilizing a typical $(T, \rho)$ tuple for the hot and dust-hostile regions of a brown dwarf atmosphere. Asymptotic analysis have been performed and one- and two-dimensional test cases have been considered applying numerical simulations in order to study the effects of turbulent disturbances of the $T-, \rho-$, and $\boldsymbol{v}$ - field on the formation of solid compounds from the gas phase.

Extending the parameter space along a typical $(T, \rho)$ trajectory of a static model atmosphere shows that dust formation at low temperatures $(T \approx 1000 \mathrm{~K})$ consumes almost instantaneously the available material until $S=1$ is reached. Here, many small particles form due to high nucleation rates. The investigations of the hotter and denser regions reveal the necessity of a cooling mechanism as it has been investigated and proposed in this paper. The higher the temperatures the larger amplitudes and wavelengths of the expansion waves are needed to create a first appropriate amount of seed nuclei. For temperatures low enough to assure the stability of the seeds, the seed particles grow to large particles since the density inside the atmosphere is higher than at higher altitudes where the temperatures are small.

Limits of stability consideration: Following a typical trajectory of a Brown Dwarf atmosphere in the $\left(T, n_{<\mathrm{H}>}\right)$ plane, high temperature compounds like $\mathrm{CaTiO}_{3}, \mathrm{Mg}_{2 x} \mathrm{Fe}_{2-2 x} \mathrm{SiO}_{4}, \mathrm{Al}_{2} \mathrm{O}_{3}$ or $\mathrm{TiO}_{2}$ may be expected to form first, if only stability arguments are considered (see also Burrows \& Sharp 1999; Lodders 2000; Chabrier et al. 2000; Leinert et al. 2000). The description of the formation of such complex particles, however, 

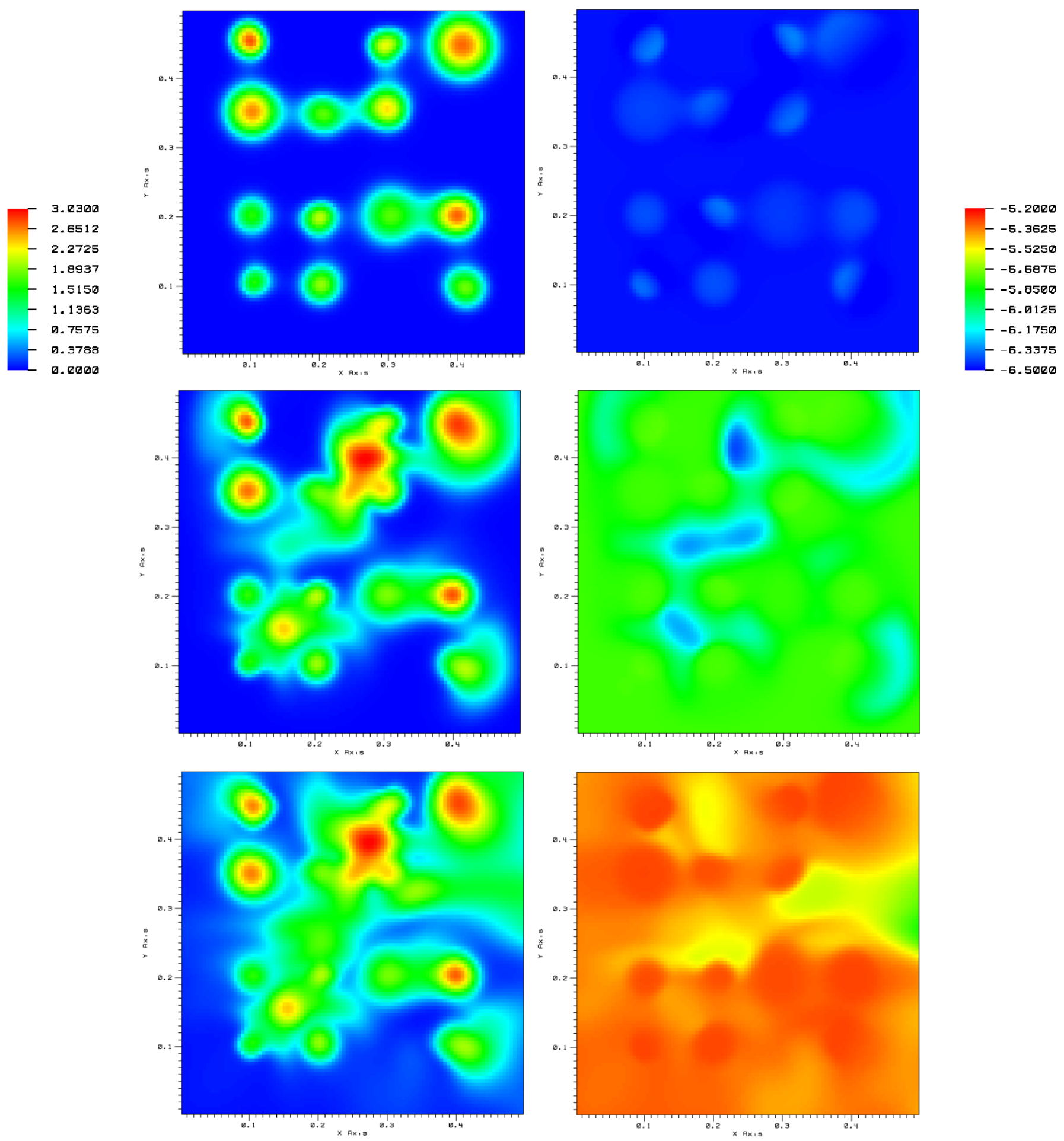

Fig. 9. Time evolution (top to bottom) of the dust particle number density $\log n_{\mathrm{d}}\left[\mathrm{cm}^{-3}\right]$ (left) and the mean particle radius $\log \langle a\rangle[\mathrm{cm}]$ (right). The color code is given left and right beside the plots. The instants of time depicted are the same like in Fig. 7. (Top: $t=3.2 \times 10^{-4} \mathrm{~s}$, middle: $t=3.3 \times 10^{-3} \mathrm{~s}$, bottom: $t=5 \times 10^{-3} \mathrm{~s}$.)

is still a matter of debate, since the nominal molecules of these solid compounds are often only present in negligible amounts (e.g. $\mathrm{Fe}_{2} \mathrm{SiO}_{4}, \mathrm{Al}_{2} \mathrm{O}_{3}$ ) or even completely absent (e.g. $\mathrm{Mg}_{2 x} \mathrm{Fe}_{2-2 x} \mathrm{SiO}_{4}, \mathrm{CaTiO}_{3}$ ) in the gas phase. Nevertheless, the rich variety of molecular species of comparable abundances in an oxygen-rich gas suggests the formation of such heterogeneous dust.

Figure 10 depicts the stability limits $S=1$ in the $\left(T, n_{<\mathrm{H}>}\right)$ plane for various oxygen bearing solid materials disregarding element depletion by dust formation 
for a solar element composition gas. Below these curves the respective solids are thermodynamically stable which suggests a "condensation" sequence ${ }^{6}$ for decreasing temperature. This conclusion is not as straightforward as it might seem. Regarding the first condensate, the stability of macroscopic dust grains is a necessary, but not a sufficient condition for the dust formation process. The formation of solid particles has to proceed via the formation of seed particles (nucleation) followed by grain growth towards macroscopic sizes (Eqs. (6), (7)). Thereby, a large supersaturation has to be achieved which results in a considerable gap between typical nucleation and stability (i.e. sublimation) temperatures of plane solids.

It has, however, been pointed out by Rossow (1978) that the supersaturation needed for nucleation might be smaller for heterogeneous nucleation processes (also refered to as chemical nucleation in Rossow 1978). Presently, heterogeneous nucleation has only been studied in carbonrich environments regarding the formation of polycyclic aromatic hydrocarbons (PAHs, Keller 1987; Goeres \& Sedlmayr 1991; Cherchneff \& Glassgold 1993) and no definite answer is available for the oxygen-rich case. However, quantum mechanical calculations performed by Patzer et al. (1998, see also Patzer et al. 1999a) have shown that homogeneous nucleation of molecular $\mathrm{Al}_{2} \mathrm{O}_{3}$ seems not to be the favorable process for the formation of corundum $\left(\left(\mathrm{Al}_{2} \mathrm{O}_{3}\right)_{N}\right)$ nuclei. If corundum nuclei form, they need to result from a heterogeneous nucleation process but no applicable description is at hand.

Candidates for the first condensate: Promising astrophysical seed candidates are titanium oxides and iron clusters, which appear in appropriate amounts in cold oxygen-rich gases; iron seeds might be even more favorable in the most dense atmospheres $\left(n_{<\mathrm{H}>}>10^{19} \mathrm{~cm}^{-3}\right)$ or in regions of high iron concentration (John \& Sedlmayr 1997). Other possible high temperature condensates, such as silicon monoxide $(\mathrm{SiO})_{N}$, are almost negligible since their nucleation rates are very low. They may rather serve as growth species than as first nuclei.

Grain size distribution: In a first study, Lüttke et al. (2000) have investigated the dust formation of a mass element moving up and falling down along a typical trajectory of a brown dwarf atmosphere model. The $\left(\mathrm{TiO}_{2}\right)_{N}$-seed particles which accumulate $\mathrm{SiO}_{2}, \mathrm{TiO}_{2}, \mathrm{Al}_{2} \mathrm{O}_{3}, \mathrm{MgO}$, and $\mathrm{Fe}$ on their way up and down through the atmosphere grow to macroscopic particle of mean sizes between $1 \ldots 3 \mu \mathrm{m}$ which is about the size we have obtained from our long term study in Sect. 4.2. Lüttke et al. (2000) - who used the same formalism to describe the dust formation process as in the present paper - calculated also the grain size distribution function $f(a)$ (compare Eq. (9)) and pointed out

\footnotetext{
${ }^{6}$ Misleading term which confuses stability and formation; stability sequence is more appropriate.
}

the size variety of the dust grains which differs widely from the interstellar power law adopted by several authors.

Burrows \& Sharp (1999) argue that dust particles in brown dwarf atmospheres should have a mean size of $\sim 0.2 \mu \mathrm{m}(\sim 0.1 \mu \mathrm{m}$ for Gl 229B in Chabrier \& Baraffe 2000 ) in order to fit the optical spectra reasonably without too much influence on the near-IR. This estimate is somewhat uncertain since also the wings of alkali resonance lines (K I, Na I) are sources of absorption in this spectral region. It is not quite clear which composition of the dust particles the two above mentioned papers had in mind. Since the composition of the grains might well depend on $T$ and $\rho$ by the stability of the compounds, differently composed grains might exhibit different sizes at different sites in the atmosphere. However, the large grains might have already gravitationally settled and only smaller particles are left floating in the atmosphere. But if one assumes that an infinite amount of time is available (as in static model atmospheres), even the smallest grains would have been gravitationally settled (rain out).

Lunine et al. (1989) argue that grains up to $100 \mu \mathrm{m}$ form by coalescence; our calculations show that they may easily be formed by single particle grow. Such large particles would even less sustain in the brown dwarf atmosphere.

Raining solids: It is now widely believed that the atmosphere of low luminosity stars like brown dwarfs are elementally depleted by the formation of dust grains which have sedimented due to the high gravity of the objects.

The sedimentation process (rain out) is known as the "drift problem" in astrophysics describing relative velocities of dust and gas due to frictional decoupling. The drifting particle will enter hotter regions where still condensible material is present without being destroyed due to the gap between the temperature of efficient nucleation and stability of the compounds. The particles will grow during their descent into the deep atmosphere if considerable material is available $(S>1)$ in this region. A competition between the increased drift velocity of the growing particle and the deceleration due to the inward decreasing gas density determines if the particle continues to fall. However, even with a very little drift velocity a static atmosphere will be completely dust free since an infinite amount of time is available in these model approaches. Since there is good observational evidence that brown dwarfs are not dust free, we conclude that brown dwarf atmospheres are not static but influenced by hydro-/thermodynamical processes, e.g. by convection. Convective material transport reaches into the atmosphere (see models by e.g. Burrows et al. 1997) and thereby replenishes these previously depleted zones. It thereby provides material for the formation of new dust. The appearance of associated acoustic waves will cause inhomogeneous distributions of dust (in size and site) in the brown dwarf atmosphere. 


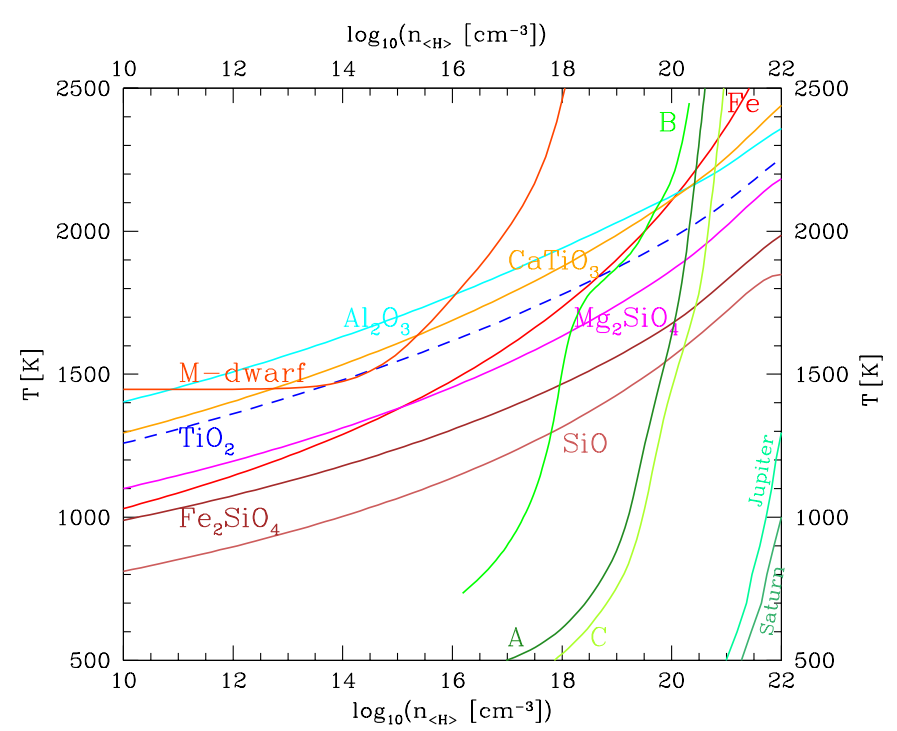

Fig. 10. Sublimation temperature $T(S=1)$ of various solid compounds as a function of the total hydrogen density $n_{<\mathrm{H}>}$ in a gas of solar abundances. Depletion by dust formation is omitted; the pure stability of possible compounds is considered. $\left(T, n_{<\mathrm{H}>}\right)$ trajectories for model atmospheres of typical M-dwarfs (Hauschildt, priv. com.), Brown Dwarfs (Tsuji et al. 1999; Model A - dust free case, Model B - dust included as opacity source, Model C - dust serves only as element sink but not as opacity source; compare also footnote 2), and gas planets (Fegley \& Lodders 1994) are superimposed.

Clouds and inhomogeneities: The results presented in this work suggest that clouds may be understood as inhomogeneous distributed dust in a brown dwarf atmosphere due to the turbulent character of its fluid field. In the terminology of Rossow (1978), these clouds would be called cooling or chemical clouds. Already small scale motions suggest the formation of cloudy inhomogeneities of the size of about $500 \mathrm{~cm}$ (compare Fig. 9, l.h.s.). The grains of such a cloud are characterized by a mean particle size $\langle a\rangle$ (compare Fig. 9, r.h.s.). Due to the time evolution of $T$ and $\rho$, particles with $a\langle\langle a\rangle$ and $a\rangle\langle a\rangle$ are formed and will therefore influence the observed spectra, too. The size of the cloud will be limited by the thermodynamic condition of the surrounding gas since a supersaturation $(S>1)$ is necessary to keep the cloud thermodynamically stable.

Where is the dust? Chabrier et al. (2000) and Els et al. (2001) show that the assumption of the existence of dust in brown dwarf atmospheres is appropriate for the interpretation of some observed $\left[M_{K}\right]-[J-K]$-colors of brown dwarfs (e.g. Kelu 1, GD 165B, LHS 102B) but not for others (Gl 229B, Gl 86B). While in the latter, the dust seems to be removed from the atmosphere out of sight by gravitational settling, dust as opacity source is needed to fit the spectra of the first examples. The existence of dust above the photosphere might indicates that dust formation is possible in regions $\tau_{\mathrm{IR}}<1$ ( $\tau_{\mathrm{IR}}-\mathrm{IR}$ optical depth), i.e. the temperature of efficient nucleation $T_{\mathrm{S}}<T\left(\tau_{\mathrm{IR}}=1\right)$. Examples are Kelu 1, GD 165B and LHS 102B. This does, however, not mean that dust and gas are in equilibrium (i.e. $S=1$ ) as stated by Allard et al. (2001) since no dust could form (see Sect. 2.1).

Gl 229B could be a brown dwarf example where dust formation might take place in layers below $\tau_{\mathrm{IR}}=1$, i.e. at $T_{\mathrm{S}}>T\left(\tau_{\mathrm{IR}}=1\right)$ inside the photosphere. Shallower regions should than already be emptied by gravitational settling. In such objects, dust formation could be influenced by convection relatively high in the atmosphere. Note that Lunine et al. (1989) show that buoyancy might be large enough to sustain convective plums of refractory condensates due to the release of latent heat. Guillot (1995), however, argue that the latent heat release of refractory materials may be too small to sustain a moist convective instability due to the small abundances of the elements involved.

Els et al. (2001) argue that the brown dwarf Gl 86B seems to fall in between the two aforementioned extreme cases. This possibly might indicate that either the dust is formed at regions $\tau_{\mathrm{IR}}=1$ or the object is observed in the state of gravitational settling when only the upper atmosphere is already dust-free.

\section{Conclusion}

Brown dwarf atmospheres are influenced by the convective interior which results in the initiation of turbulent motions and thereby in the creation of acoustic waves. The existence of a non-static fluid field can also be inferred from the indirect observation of dust in brown dwarfs like Kelu 1 since all the dust would have settled and thereby removed from the atmosphere in a static situation.

The study of the interaction of dust formation and turbulent motion has been restricted to the microscopic scale regime in this paper since only here a direct simulation of the model equations (hydro-/thermodynamics, dust formation, element conservation) is possible. For any increase of the scale regime a model for the smallest scales will be needed in order to properly take into account all scales which influence the system. Our work involves:

- Asymptotic analysis to study the threshold characteristics of the dust complex and the long-term behavior of the dust forming system. A test problem has been set up and very good agreement with the numerical solution of the full model has been achieved;

- Numerical simulations of the full model problem in order to study self-consistently the interaction between turbulence and dust formation in $1 \mathrm{D}$ and in $2 \mathrm{D}$.

The study of the $1 \mathrm{D}$ problems has revealed the complex interactions between dust formation, expansion waves created by turbulent motions and the radiative cooling process:

- Interacting acoustic expansion waves can cause a temperature decrease low enough to initiate dust nucleation for a short time interval. The seed particles grow until they reach a size where the dust opacity is large 
enough to accelerate radiative cooling. Dust nucleation is henceforth re-initiated which causes a further intensified radiative cooling which again increases the nucleation rate and the amount of dust particles formed. This run-away process is stopped if either the radiative equilibrium temperature of the gas is reached or all condensible material is consumed. Meanwhile, the seed particles grow to macroscopic sizes;

- Expansion waves are identified as physical mechanism to form locally large amounts of dust in an otherwise hostile environment for a solid component.

The study of the $2 \mathrm{D}$ problem has confirmed the potential of acoustic waves to initiate dust formation. An additional mechanism for the generation of inhomogeneous dust distributions was observed:

- Randomly moving acoustic waves have been shown to also create temperature increases. Dust nucleation is thereby interrupted in regions where it previously was possible. Therefore, interacting acoustic waves might cause an intermittent distribution of dust in regions where dust might: Command not found

formation initially took place.

The results presented in this work suggest that clouds may be understood as inhomogeneous distributed dust in a brown dwarf atmosphere due to the turbulent character of its flow field.

Regarding the dust to be formed under conditions typical for a brown dwarf atmosphere we have worked out that

- stability sequences are not sufficient to decide which species nucleates first since a large supersaturation has to be achieved causing a gap between nucleation and sublimation temperatures;

- particles sizes are related to the number density of the particles. The number of particles depends on the thermodynamical conditions of their site of formation.

The complete understanding of the recently observed nonperiodic variability of brown dwarfs needs further improvements of the model(s) presented so far. This concerns the microphysics (e.g. chemistry) as well as the macrophysics which determines the large scale flow pattern of the atmosphere. A natural next step of our own work would be an extension of the model scales into the mesoscopic regime.

Acknowledgements. The referee is thanked for the useful advises to the manuscript. We thank Prof. T. Tsuji for providing us with his $1 \mathrm{D}$ models of static model atmospheres of brown dwarfs. Discussions and valuable comments on the manuscript by Dr. P. Woitke and Dr. C. Bailer-Jones are greatly acknowledged. We thank Prof. U. G. Jørgensen for the careful reading of the manuscript. Dipl.-Ing. H. Schmidt is thanked for providing his hydro-code and for the support in setting up the computations. This work has been supported by the $D F G$ (grants SE 420/19-1, Kl 611/7-1, Kl 611/9-1).

\section{References}

Allard, F., Hauschildt, P., Alexander, D., Tamanai, A., \& Schweitzer, A. 2001, ApJ, in press

Allard, F., Hauschildt, P., \& Schweitzer, A. 2000, ApJ, 539, 366

Asplund, M., Nordlund, Å., Trampedach, R., Allende Prieto, C., \& Stein, R. 2000, A\&A, 359, 729

Bailer-Jones, C. A. L., \& Mundt, R. 1999, A\&A, 348, 800

Bailer-Jones, C. A. L., \& Mundt, R. 2001a, A\&A, 367, 218

Bailer-Jones, C. A. L., \& Mundt, R. 2001b, A\&A, submitted

Bender, C. M., \& Orszag, S. A. 1999, Advanced Mathematical Methods for Scientists and Engineers (Springer, New York, Berlin, Heidelberg)

Burrows, A., \& Sharp, C. M. 1999, ApJ, 512, 843

Burrows, A., Marley, M., Hubbard, W. B., Lunine, J. I., Guillot, T., et al. 1997, ApJ, 491, 856

Chabrier, G., \& Baraffe, I. 1997, A\&A, 327, 1039

Chabrier, G., \& Baraffe, I. 2000, A\&AR, 38, 337

Chabrier, G., Baraffe, I., Allard, F., \& Hauschildt, P. 2000, ApJ, 542, 464

Chase, Jr. M. W., Davies, C. A., Downey, Jr. J. R., et al. 1985, in J. Phys. Chem. Ref. Dat., 14 Suppl. 1, National Bureau of Standards

Cherchneff, I., \& Glassgold, A. E. 1993, ApJ, 419, L41

Dominik, C., Gail, H.-P., Sedlmayr, E., \& Winters, J. M. 1990, A\&A, 240, 365

Dominik, C., Sedlmayr, E., \& Gail, H.-P. 1993, A\&A, 277, 578

Draine, B. T., \& Lee, H. M. 1984, ApJ, 285, 89

Els, S., Sterzki, M., Marchis, F., et al. 2001, A\&A, 370, L1

Fegley, B., \& Lodders, K. 1992, Icarus, 110, 117

Fleischer, A. J., Gauger, A., \& Sedlmayr, E. 1992, A\&A, 266, 321

Frisch, U. 1995, Turbulence (Cambridge University Press, Cambridge)

Gail, H.-P., \& Sedlmayr, E. 1986, A\&A, 166, 225

Gail, H.-P., \& Sedlmayr, E. 1987, A\&A, 171, 197

Gail, H.-P., \& Sedlmayr, E. 1988, A\&A, 206, 153

Gail, H.-P., \& Sedlmayr, E. 1999, A\&A, 347, 594

Gail, H.-P., Keller, R., \& Sedlmayr, E. 1984, A\&A, 133, 320

Gelino, C. R., Marley, M. S., \& Holtzman, J. A. 2001, ApJ, in press

Goeres, A., \& Sedlmayr, E. 1991, Chem. Phys. Lett., 184(4), 310

Grossman, L. 1972, Geochim. Cosmochim. Acta, 38, 47

Guillot, T. 1995, Science, 269, 1697

Helling, Ch., Lüttke, M., Sedlmayr, E., \& Klein, R. 2000, In Warnecke, Hyperbolic Problems: Theory - Numeric Applications, Otto-von-Guericke Universität Magdeburg, in press (http://astro.physik.tu-berlin.de/ 'chris/)

Helling, Ch., Winters, J. M., \& Sedlmayr, E. 2000, A\&A, 358, 651

Höfner, S., Dorfi, E. A. 1992, A\&A, 265, 207

Jeong, K. S. 2000, Dust shells around oxygen-rich Miras and long-period variables, Ph.D. Thesis (Technische Universität, Berlin, Germany)

Jeong, K. S., Chang, C., Sedlmayr, E., \& Sülzle, D. 2000, J. Phys. B, 33, 3417

John, M., \& Sedlmayr, E. 1997, Astrophys. Space Sci., 251(1-2), 219

Jones, H., \& Tsuji, T. 1997, AJ, 480, L39

Jones, H. R. A., Longmore, A. J., Jameson, R. F., \& Mountain, C. M. 1994, MNRAS, 267, 413 
Keller, R. 1987, in Léger A., d'Hendecourt L., Boccara N., Polycyclic Aromatic Hydrocarbons and Astrophysics, 387 (Reidel Publ. Comp.)

Kirkpatrick, J. D., Allard, F., Bida, T., et al. 1999, ApJ, 519, 834

Klein, R. 1995, JCP, 121, 213

Leinert, C., Allard, F., Richichi, A., \& Hauschildt, P. 2000, A\&A, 353, 691

Liebert, J., Reid, I., Burrows, A., et al. 2000, AJ, 533, L155

Lodders, K. 1999, ApJ, 519, 793

Lunine, J., Hubbard, W., Burrows, A., Wang, Y.-P., \& Garlow, K. 1989, ApJ, 338, 314

Lüttke, M., Helling, Ch., John, M., Jeong, K., Woitke, P., et al. 2000, AG Abstr. Ser., 17, 52

(http:// astro.physik.tu-berlin.de/Publikationen/ Publ00/index.html)

Martín, E., Zapatero Osorio, M., \& Lehto, H. 2001, ApJ, in press

Noll, K. S., Geballe, T. R., \& Marley, M. S. 1997, ApJ, 489, L87

Oppenheimer, B., Kulkarni, S., Matthews, K., \& van Kerkwijk, M. H. 1998, ApJ, 502, 932

Patzer, A., Chang, C., John, M., \& Sedlmayr, E. 1998, in ed. T. Le Bertre, A. Lèbre, \& C. Waelkens, IAU Symp. 191: AGB stars, Poster P3-02. ADS
Patzer, A. B. C., Chang, C., Sedlmayr, E., \& Sülzle, D. 1999a, Eur. Phys. J. D, 6, 57

Patzer, A. B. C., Helling, Ch., Winters, J. M., \& Sedlmayr, E. 1999b, AG Abstr. Ser., 15, 107

Pavlenko, Y., Zapatero Osorio, M., \& Rebolo, R. 2000, A\&A, 355,245

Rossow, W. 1978, Icarus, 36, 1

Schneider, T., Botta, N., Geratz, K., \& Klein, R. 1999, JCP, 155,248

Smiljanovski, V., Moser, V., \& Klein, R. 1997, Comb. Theory Modell., 1, 183

Stevenson, D. 1986, Astrophysics of brown dwarf, 218 (Cambridge University Press)

Tinney, C. G., Reid, I. N., \& Mould, J. 1993, AJ, 414(1), 254

Tsuji, T., Ohnaka, K., \& Aoki, W. 1996, A\&A, 305, L1

Tsuji, T., Ohnaka, K., Aoki, W., \& Nakajima, T. 1996, A\&A, 308, L29

Tsuji, T., Ohnaka, K., \& Aoki, W. 1999, ApJ, 520, L119

Winters, J. M., Le Bertre, T., Jeong, K. S., Helling, Ch., \& Sedlmayr, E. 2000, A\&A, 361, 641

Zöckler, M. 1997, Master's Thesis (Technische Universität Berlin, Germany) 